
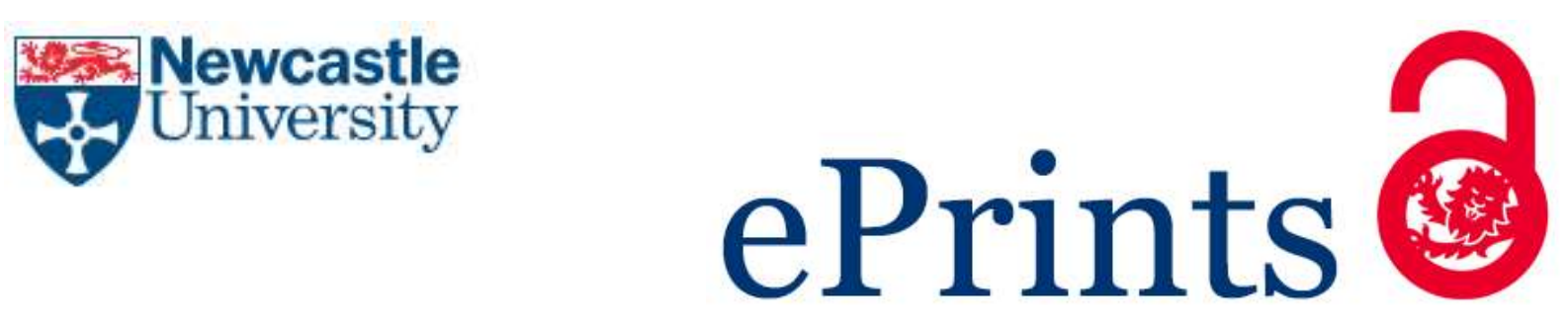

Liang Y, Tao L. Interaction of vortex shedding processes on flow over a deepdraft semi-submersible. Ocean Engineering 2017, 141, 427-449.

\title{
Copyright:
}

(C) 2017. This manuscript version is made available under the CC-BY-NC-ND 4.0 license

DOI link to article:

https://doi.org/10.1016/j.oceaneng.2017.06.056

Date deposited:

$25 / 07 / 2017$

Embargo release date:

05 July 2018

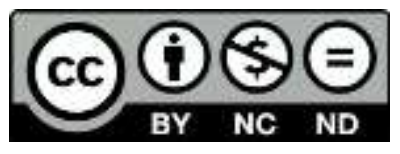

This work is licensed under a

Creative Commons Attribution-NonCommercial-NoDerivatives 4.0 International licence 


\section{Interaction of Vortex Shedding Processes on Flow over a 2 Deep-Draft Semi-Submersible}

3 Yibo Liang, Longbin Tao*

4 School of Marine Science and Technology, Newcastle University, Newcastle upon Tyne, NE1 7RU, 5 UK

\section{Abstract}

7 A numerical study on the flow over a Deep-Draft Semi-Submersible (DDS) for both stationary and

8 Vortex-Induced Motions (VIM) was carried out using the Computational Fluid Dynamics (CFD), with

9 the aim to investigate the overall hydrodynamics of the structure. In order to study the fluid physics

10 associated with VIM, a comprehensive numerical simulation was conducted to examine the

11 characteristics of vortex formations, shedding processes and especially their interactions due to the multiple cylindrical columns. In addition to the vortex shedding characteristics, the drag and lift

13 forces on each member of the overall structure were calculated. It is revealed that under $45^{\circ}$

14 incidence, the transverse forces induced by the portside and starboard side columns are the dominant

15 excitation forces responsible to VIM while the horizontal member - pontoons restraining VIM. In

16 addition, the hysteresis phenomenon observed between the force and motion domains - the peak lift

17 force occurs slightly earlier than the peak transverse motion is mainly due to the vortices shed from the upstream column move back to impinge on one of the side columns after impinging on the other

19 side column and the symmetrical strong vortices which shed from the side columns.

\section{Keywords}

\footnotetext{
${ }^{*}$ Corresponding author. Tel.: +44 (0) 191208 6670; Fax: +44 (0) 191208 5491; E-mail address: longbin.tao@newcastle.ac.uk
} 
21 Vortex-Induced Motions (VIM); Deep-Draft Semi-Submersible (DDS); Computational Fluid

22 Dynamics (CFD)

\section{Nomenclature}

$24 \quad A_{x} / L \quad$ Non-dimensional characteristics amplitude of in-line motion

$25 A_{y} / L \quad$ Non-dimensional characteristics amplitude of transverse motion

$26 \quad B_{L} \quad$ Platform width

$27 \quad B_{T} \quad$ Platform draft

$28 \quad C_{a} \quad$ Added mass coefficient

$29 C_{D} \quad$ Drag force coefficient

$30 C_{L} \quad$ Lift force coefficient

$31 \quad D \quad$ Column projected length

$32 f \quad$ Vortex shedding frequency

$33 f_{0} \quad$ Natural frequency in still water

$34 \quad \mathrm{Fr} \quad$ Froude number

$35 \quad F_{D} \quad$ Hydrodynamic drag force acting on the structure

$36 F_{L}, F_{y}$ Hydrodynamic lift force acting on the structure

$37 \quad G C I \quad$ Grid convergence index

$38 H \quad$ Immersed column height above the pontoon

$39 L \quad$ Column width

$40 \quad m \quad$ Platform mass

$41 \quad m_{a} \quad$ Added mass

$42 \quad P \quad$ Pontoon height

43 Re Reynolds number

44 rms Root mean square

$45 \quad S \quad$ Distance between centre columns

46 St Strouhal number

$47 \quad T_{0} \quad$ Natural periods in still water

$48 \Delta t \quad$ Numerical simulation time step

$49 U, U_{c} \quad$ Current speed

$50 \quad U r \quad$ Reduced velocity

$51 \rho \quad$ Fresh water density 
$52 \Delta \quad$ Displacement

$53 \lambda \quad$ Scale ratio

$54 \vec{\omega}_{x} \quad$ Streamwise vorticity

$55 \vec{\omega}_{y} \quad$ Transverse vorticity

$56 \quad \vec{\omega}_{z} \quad$ Spanwise vorticity

$57 \quad X \quad$ In-line motion

$58 \quad Y \quad$ Transverse motion

$59 y^{+} \quad$ Y plus value

\section{1. Introduction}

61 Vortex-Induced Motions (VIM) have been receiving continuous attention in the field of offshore exploration and exploitation as an increasing number of deep-draft floating structures have been operating in different regions around world. Deep-draft floating structures are well known for their

64 favourable vertical motions behaviour compared with other types of floating offshore structures.

65 However, the increases in the structure's draft can also lead to more severe VIM, which may lead to

66 potential damage particularly causing fatigue to the mooring and riser systems.

67 VIM have often been observed since the Genesis Spar platform was commissioned in 1997 (Fujarra et al., 2012; Kokkinis et al., 2004). Rijken and Leverette (2009) reported VIM phenomenon on a semisubmersible in field measurements. Ma et al. (2013) also observed the presence of VIM from recent field measurements. In this aspect, a number of studies on the VIM behaviours have been carried out, including both experimental and numerical studies. On the experimental investigation side, as pointed out by Fujarra et al. (2012) in their comprehensive review, VIM are now much better understood. However, it is still lack of understanding about the VIM mechanism on multiple cylindrical structures such as the semi-submersible and the Tension-Leg Platform (TLP). The vortex shedding processes and subsequent VIM are much more complex than those of single cylindrical structures due to the multi-columns, pontoons and their interactions with the vortex shedding processes.

Waals et al. (2007) conducted several VIM tests on both DDS and TLP to examine the influences of mass ratio and draft effects. A series of model tests on a DDS were carried out by Hong et al. (2008), 
and the results showed that under a strong current, the DDS will have more significant VIM responses compared with the wave-current coupling condition. Rijken and Leverette (2008) experimentally studied the VIM responses of a DDS, and observed that wave and external damping can affect the VIM responses. Through their tests, it was noted that the relatively low sea states do not particularly influence the VIM responses under the so-called "lock-in" region. Moreover, the additional damping delayed the onset of VIM to a higher reduced velocity. Rijken et al. (2011) analysed the influences of SCR systems and appurtenances on VIM for a DDS. Their work showed that the appurtenances on the vertical faces of the columns and above the pontoon can alter the VIM responses. Gonçalves et al. (2012) subsequently investigated the effects of the current angle and appendages on a conventional semi-submersible. The presence of VIM on a conventional semi-submersible has been confirmed in their works. Following on from their initial outcomes, Gonçalves et al. (2013) further studied other relevant factors such as the draft conditions, the external damping and wave effects on VIM developing by performing a series of towing tank tests. Additionally, Gonçalves et al. (2015) performed experimental tests focusing on the effects of different column designs on the VIM responses. The results showed that the circle section shaped column design has the most severe transverse motions at 0 degree flow incidence and that the square section shaped column design has the most significant transverse motion at 45 degree flow incidence. Recently, Antony et al. (2016) studied the effects of damping on VIM and investigated the force distribution on each member of the structure in detail by an experimental routine. The work done by each member was presented in their investigations. The investigations showed that for 45 degree flow incidence, when the maximum transverse VIM response occurs, three upstream columns excited VIM. The horizontal member pontoons, however, were noted to limit the VIM responses.

In the last decade, the continued technological advances offer ever-increasing computational power, in which CFD methods are rapidly gaining popularity for VIM predictions. Lefevre et al. (2013) proposed the guidelines for undertaking the Spar VIM simulations. Tan et al. (2013) performed a series of CFD simulations for VIM on a multi-column floater. Lee et al. (2014) studied the differences 
numerically and experimentally investigated the VIM responses of a deep-draft column stabilized

107 floater. Their work shows that the damping effects of the riser and mooring systems are very important in CFD simulations. Vinayan et al. (2015) increased the confidence for CFD simulations on the VIM predictions of a deep-draft column stabilized floater through a series of numerical simulations on a PC-semi with different drafts and arrangements. Liu et al. (2015) numerically investigated the effects of pontoon on hydrodynamic forces for a stationary DDS model and revealed that the DDS with the different numbers of pontoons affects both drag and lift forces on the stationary

113 structures. Koop et al. (2016) carried out a series of CFD studies to illustrate the results of the scale

114 and damping effects for VIM on a semi-submersible. Their work showed that the scale effects at 45

115 degree incidence are less than that at 0 degree incidence. Under 45 degree incidence, the VIM 116 response at prototype Reynolds number is found to be similar compared with that at model scale

117 Reynolds number. Similar observation was also reported by Lee et al. (2014).

\section{Numerical simulation}

\subsection{Computational overview}

120 A comprehensive numerical study was conducted in this section, with the aim to examine the vortex

121 shedding characteristics and the associated fluid dynamics. The numerical schemes are introduced and followed by a sensitivity assessment in order to perform a computationally efficient numerical analysis.

124 The Detached Eddy Simulation (DES) method was used in this study. For the DES model, the 125 Improved Delayed Detached Eddy Simulation (IDDES) model (Shur et al., 2008) with the Spalart126 Almaras (SA) (Spalart et al., 1997) was used. All the simulations were carried out by using a Star$127 \mathrm{CCM}+9$ package. The principle dimensions of the deep-draft semi-submersible analysed in this section are given in Table 1. Two models with different scale ratios are simulated in the present study, with resulting flow 
conditions ranging from Reynolds number $3 \times 10^{5}$ to $1.1 \times 10^{6}$. Model I is used for the stationary structure simulations where the model scale ratio is 1:128. Model II is for the VIM simulations where the model scale ratio is 1:64. For all simulations, the computational domain $9 B_{L} \times 6 B_{L} \times 3 B_{T}$ is used (where $B_{L}$ is the overall hull width of the semi-submersible and $B_{T}$ is the draft of the semidirection.

A)

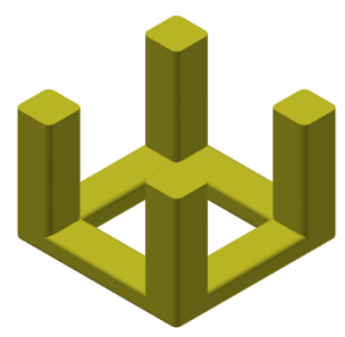

B)

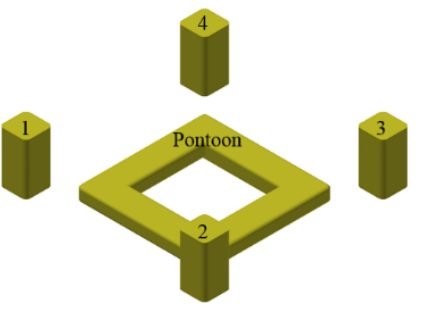

Fig. 1. The DDS model (A is the entire model and B is the decomposed model which show the definition of the individual members). scale ratio as 1:128, and the model II is the VIM model which presents scale ratio as 1:64). 


\begin{tabular}{lccc}
\hline & $\begin{array}{c}\text { Prototype } \\
(\mathbf{m})\end{array}$ & $\begin{array}{c}\text { Model I } \\
(\mathbf{m})\end{array}$ & $\begin{array}{c}\text { Model II } \\
(\mathbf{m})\end{array}$ \\
\hline Distance between centre columns $(\boldsymbol{S})$ & 72.5 & 0.567 & 1.133 \\
Column width $(\boldsymbol{L})$ & 19.5 & 0.152 & 0.305 \\
Immersed column height above the pontoon $(\boldsymbol{H})$ & 37.0 & 0.289 & 0.578 \\
Pontoon height $(\boldsymbol{P})$ & 10.0 & 0.078 & 0.156 \\
\hline
\end{tabular}

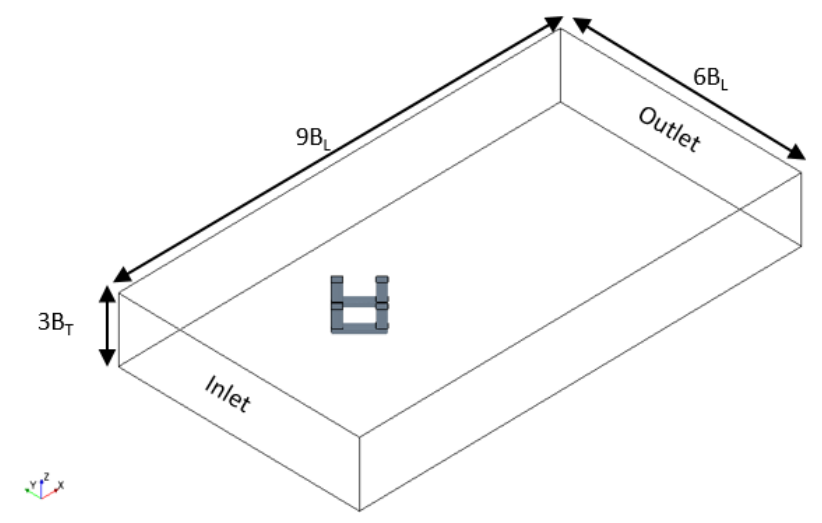

147 Fig. 2. Computational domain.

The polyhedral mesh (CD-adapco, 2014) was used in the present study. The overall elements mesh is shown at a middle-depth horizontal layer in Fig. 3. In the present study, a near wall refinement method named "Prism Layer Mesher" is adopted. The $y^{+}$values are smaller than 1 in all simulations, where $y^{+}=u * \Delta y_{1} / v\left(u *\right.$ denotes the friction velocity at the nearest wall, $\Delta y_{1}$ is the first layer thickness and $v$ is the kinematic viscosity). Other five regional refinements are carried out in the domain to refine both the near wake and the far wake region (see Fig. 4).

The boundary conditions are kept all the same in the present study. At the inlet, a uniform and constant velocity is specified directly for all sensitivity studies. The pressure at boundary was extrapolated from the adjacent cell using reconstruction gradients (CD-adapco, 2014). Along the outlet boundary, the pressure is prescribed to be equal to zero. The velocity at the boundary was extrapolated from the interior using reconstruction gradients (CD-adapco, 2014). For the body surface of the deep-draft semi-submersible, a no-slip boundary condition was specified in terms of the tangential velocity which is explicitly set to be zero and the pressure at the boundary was extrapolated from the adjacent cells using reconstruction gradients (CD-adapco, 2014). As the Froude number is 

quite small $(F r<0.2)$ in all simulations, the free surface effects can be ignored and the free surface boundary condition is prescribed as being a symmetry boundary.

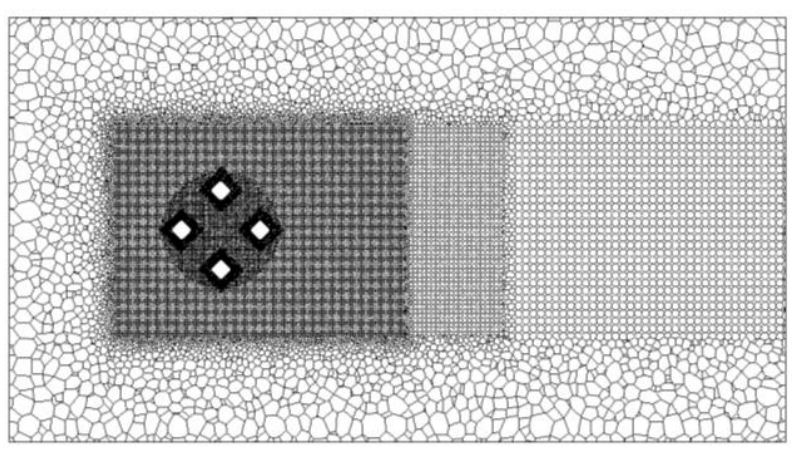

165

166

167

168

Fig. 3. Visualisation of the mesh at the middle draft level of the DDS ( $X Y$ plane at the middle draft of the DDS).

\subsection{Sensitivity studies}

In order to investigate the numerical mesh sensitivity of the calculated results, a mesh sensitivity study had been carried out with different levels of refinement grids resolution following the guideline proposed by Celik et al. (2008). The Reynolds number set for the mesh sensitivity study is $1.1 \times 10^{6}$, which is the highest Reynolds number in all the undertaken simulations. The details of the mesh sensitivity studies are shown in Table 2. Results for all cases are obtained by averaging after more than fifteen vortex shedding cycles.

Table 2. Calculations of discretization error (Celik et al., 2008); GCI index represents the numerical uncertainty.

\begin{tabular}{cccc}
\hline & $\overline{\boldsymbol{C}}_{\boldsymbol{D}}$ & $\boldsymbol{C}_{\boldsymbol{L r m s}}$ & $\boldsymbol{S t}$ \\
\hline $\begin{array}{c}\boldsymbol{N}_{\boldsymbol{l}}, \boldsymbol{N}_{\mathbf{2}}, \boldsymbol{N}_{3} \\
\text { (thousand) }\end{array}$ & $6860,3430,937$ & $6860,3430,937$ & $6860,3430,937$ \\
$\boldsymbol{r}_{\mathbf{2 1}}$ & 1.333 & & \\
$\boldsymbol{r}_{32}$ & 1.6 & 1.333 & 1.333 \\
$\boldsymbol{\emptyset}_{\boldsymbol{1}}$ & 1.066 & 1.6 & 1.6 \\
$\boldsymbol{\emptyset}_{2}$ & 1.068 & 0.093 & 0.131 \\
$\boldsymbol{\emptyset}_{3}$ & 1.053 & 0.101 & 0.131 \\
$\boldsymbol{p}$ & 4.561 & 0.139 & 0.134 \\
\end{tabular}




\begin{tabular}{cccc}
\hline $\boldsymbol{G C I}_{\text {normal }}^{32}$ & $0.23 \%$ & $18.31 \%$ & $\mathrm{NaN}$ \\
$\boldsymbol{G C I}_{\text {fine }}^{21}$ & $0.09 \%$ & $9.13 \%$ & $\mathrm{NaN}$ \\
\hline
\end{tabular}

$S t=\frac{f L}{U_{c}}$

Following the guideline proposed by Celik et al. (2008), $N_{3}, N_{2}, N_{1}$ represent the total number of grids from a course grid refinement level to a relatively fine grid refinement; $r$ is the grid refinement factor, where $r=h_{\text {coarse }} / h_{\text {fine }}$ and $h$ is the grid size; $\varnothing$ is the calculation results for different grid refinements; $p$ is the apparent order; $G C I$ is the grid convergence index which shows the level of numerical uncertainty. The resulting force coefficients $\left(C_{D}, C_{L}\right)$ and the Strouhal number $(S t)$ which are compared in the sensitivity studies are defined as:

$C_{D}=\frac{F_{D}}{\frac{1}{2} \rho U_{C}^{2} A}$

$C_{L}=\frac{F_{L}}{\frac{1}{2} \rho U_{C}^{2} A}$

where, $F_{D}$ is the drag force on the structure, $F_{L}$ is the lift force on the structure, $\rho$ is the fresh water density, $U_{C}$ is the free stream velocity, $A$ is the projected area of the immersed structure, $f$ is the vortex shedding frequency obtained from the power spectra of lift force coefficient fluctuations as followed by Schewe (1983) and $L$ is the width of the DDS column.

As shown in Table 2, there is a reduction in the $G C I$ index for the successive finer grid refinements, where $G C I_{\text {fine }}^{21}$ is less than $G C I_{\text {normal }}^{32}$. The $G C I$ index for the fine grid refinement $\left(G C I_{\text {fine }}^{21}\right)$ is relatively low compared to the coarse level of grid refinement $\left(G C I_{\text {normal }}^{32}\right)$, indicating that the dependence of the numerical simulation on the mesh has been reduced. As the $G C I$ index reduction from the coarse grid refinement to the fine grid refinement is relatively high, then the mesh convergence (grid independent) can be said to have been nearly achieved. Additionally, as the St may indicate that the "exact" solution has been attained, in this case, calculations with additional grid refinements are 
performed in Fig. 4. It shows that the $S t$ is converged around the value of 0.131 . Therefore, the numerical uncertainty for the Strouhal number is shown as "NaN" in Table 2. These mesh sensitivity studies show that the $N_{2}$ numerical mesh setting is fine enough to obtain reliable results with an acceptable computation time and they are used in further numerical studies.

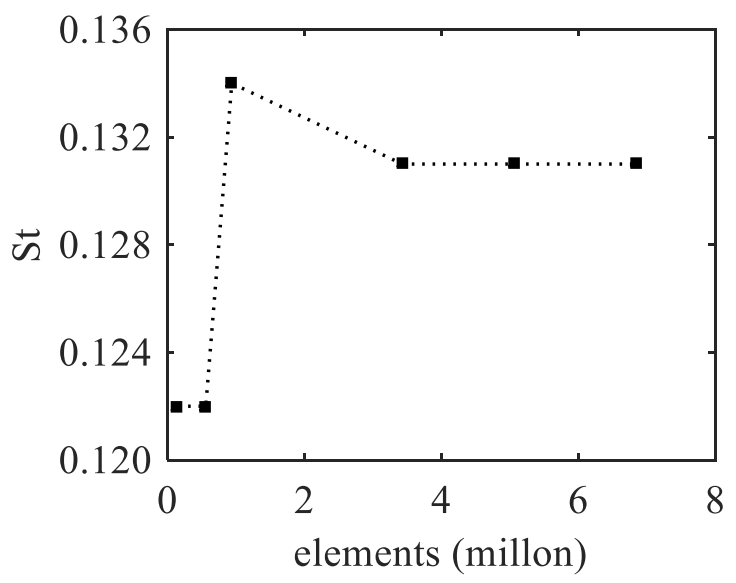

Fig. 4. Calculations with additional grid refinements for the Strouhal number $(S t)$.

The non-dimensional time step is chosen as 0.008 (non-dimensional time step $=\Delta t U / L$, where $\Delta t$ is the time step, $U$ is the inlet velocity and $L$ is the width of the DDS column) for all cases based on sensitivity test. The constant non-dimensional time step size will result in varying courant (CFL) numbers as the grid is refined. A major benefit of employing the IDDES approach is that a large portion of the flow should be resolved with the Large Eddy Simulation (LES), but this requires rather strict CFL number limits. In the present study, the CFL numbers for the majority of the overall flow region are less than 1 . Only in some tiny flow areas, the CFL numbers are found to be between 1 to 2 . Therefore, the time step is considered to be fine enough for the current simulations' requirement (Liang et al., 2016).

\subsection{Reduced velocity}

When discussing VIM, the so-called reduced velocity $(U r)$ is usually used as the reference value. The reduced velocity $(U r)$ is defined as: 
$214 U r=\frac{U T_{0}}{D}$,

215 where $U$ is the current speed, $T_{0}$ is the natural period of the motions in calm water and $D$ is the

216 projected length of the column.

\section{3. Results and discussion}

218 Two different conditions (stationary and VIM) of a typical deep-draft semi-submersible under 45

219 degree flow incidence were investigated using the present numerical model and their results are

220 further compared with the experimental data from model tests conducted in a circulating water

221 channel and a towing tank respectively.

222 The results from both previous works and present outcomes are summarised in Table 3. As confirmed in both field measurements and model tests (Gonçalves et al., 2012; Koop et al., 2016; Lee et al., 2014; Ma et al., 2013; Magee et al., 2011; Rijken and Leverette, 2008; Waals et al., 2007), for square section shaped multi-columns structures, the most severe transverse motion occurred at 45 degree incidence. As Koop et al. (2016) noted, the scale effects of a DDS in a 45 degree flow are less than that in a 0 degree flow. This indicates that the Reynolds number does not have a large effect on the model predictions at 45 degree incidence. Aiming to investigate the VIM of a DDS at a realistic field condition with the real engineering applications, the flow over a stationary structure and a VIM model of a DDS at 45 degree incidence have been numerically investigated after a rigorous validation against the experimental data. The hydrodynamic loads on different members of the structure, such as four columns and pontoons, are compared in order to quantify the determining factors which induced

233 VIM excitation. Moreover, the flow patterns are further examined to reveal the insights of the vortex 234 dynamics associated with VIM. structures. 


\begin{tabular}{lccccc}
\hline & $\boldsymbol{\lambda}$ & $\boldsymbol{H} / \boldsymbol{L}$ & $\boldsymbol{U} \boldsymbol{r}$ & $\boldsymbol{m a x} \boldsymbol{A}_{\boldsymbol{y}} / \boldsymbol{L}$ at $\mathbf{0}^{\circ}$ & $\max \boldsymbol{A}_{\boldsymbol{y}} / \boldsymbol{L}$ at $\mathbf{4 5}^{\circ}$ \\
\hline Waals et al. (2007) & $1: 70$ & 1.75 & $4.0 \sim 40.0$ & -- & 0.320 \\
Rijken and Leverette (2008) & $1: 50$ & 2.18 & $1.0 \sim 15.0$ & 0.151 & 0.468 \\
Magee et al. (2011) & $1: 70$ & 1.50 & $4.0 \sim 13.0$ & 0.269 & 0.319 \\
Gonçalves et al. (2012) & $1: 100$ & 1.14 & $2.5 \sim 20.0$ & 0.268 & 0.382 \\
Ma et al. (2013) & $1: 1$ & -- & $3.2 \sim 13.7$ & 0.163 & 0.218 \\
Lee et al. (2014) & $1: 67$ & 1.78 & $4.0 \sim 20.0$ & -- & 0.393 \\
Lee et al. (2014) & $1: 1$ & 1.78 & $4.0 \sim 20.0$ & -- & 0.344 \\
Koop et al. (2016) & $1: 54$ & -- & $3.0 \sim 10.0$ & -- & 0.470 \\
Present work & $1: 64$ & 1.90 & $3.4 \sim 14.1$ & 0.279 & 0.742 \\
\hline
\end{tabular}

\section{1. "lock-in" phenomenon}

239 The "lock-in" phenomenon is defined as being the synchronized oscillation region that is experienced

240 as VIM develops. When flow over a bluff body, vortices are generated on the downstream area of the

241 structure which are detached periodically and alternately from each opposite sides of the structure.

242 The structure affected by the vortex shedding may thus begin oscillating either side to side or in a fore

243 and aft manner. If the vortex shedding frequency closes to the natural frequency of the structure, the

244 motion can be amplified. This amplification phenomenon is named as the "lock-in". The "lock-in"

245 always happened at reduced velocity around seven (See Fig. 5). It can be clearly seen in Fig. 5 that the

246 structure experiences the largest transverse motions at 45 degree incidence. Fig. 6 shows the spanwise

247 vorticity contour $\left(\vec{\omega}_{z} D / U\right)$ when the "lock-in" generated. Under 45 degree incidence, the non-

248 dimensional spanwise vorticity is stronger than that under 0 degree incidence. In the present

249 investigation, the hydrodynamic loads on the structures and the vortex shedding interactions under 45

250 degree incidence are analysed and discussed. 


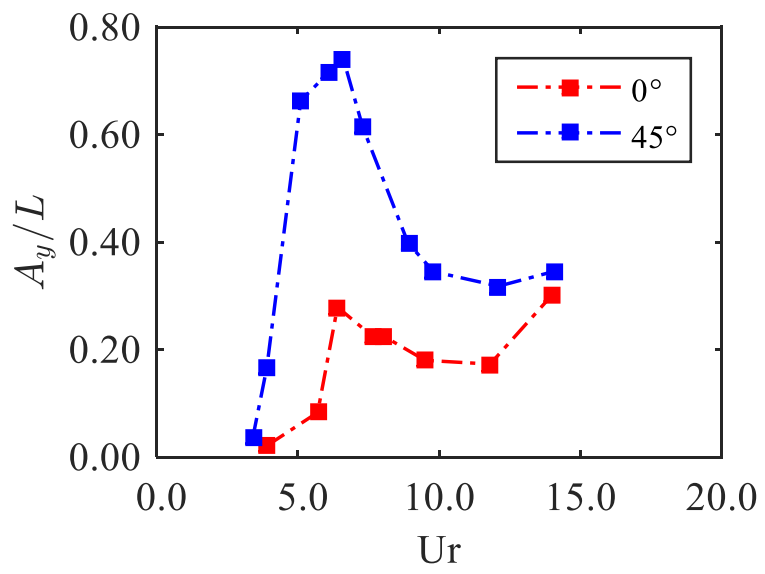

252 Fig. 5. Non-dimensional transverse characteristic amplitudes $\left(A_{y} / L\right)$ obtained from the present towing 253 tank test.

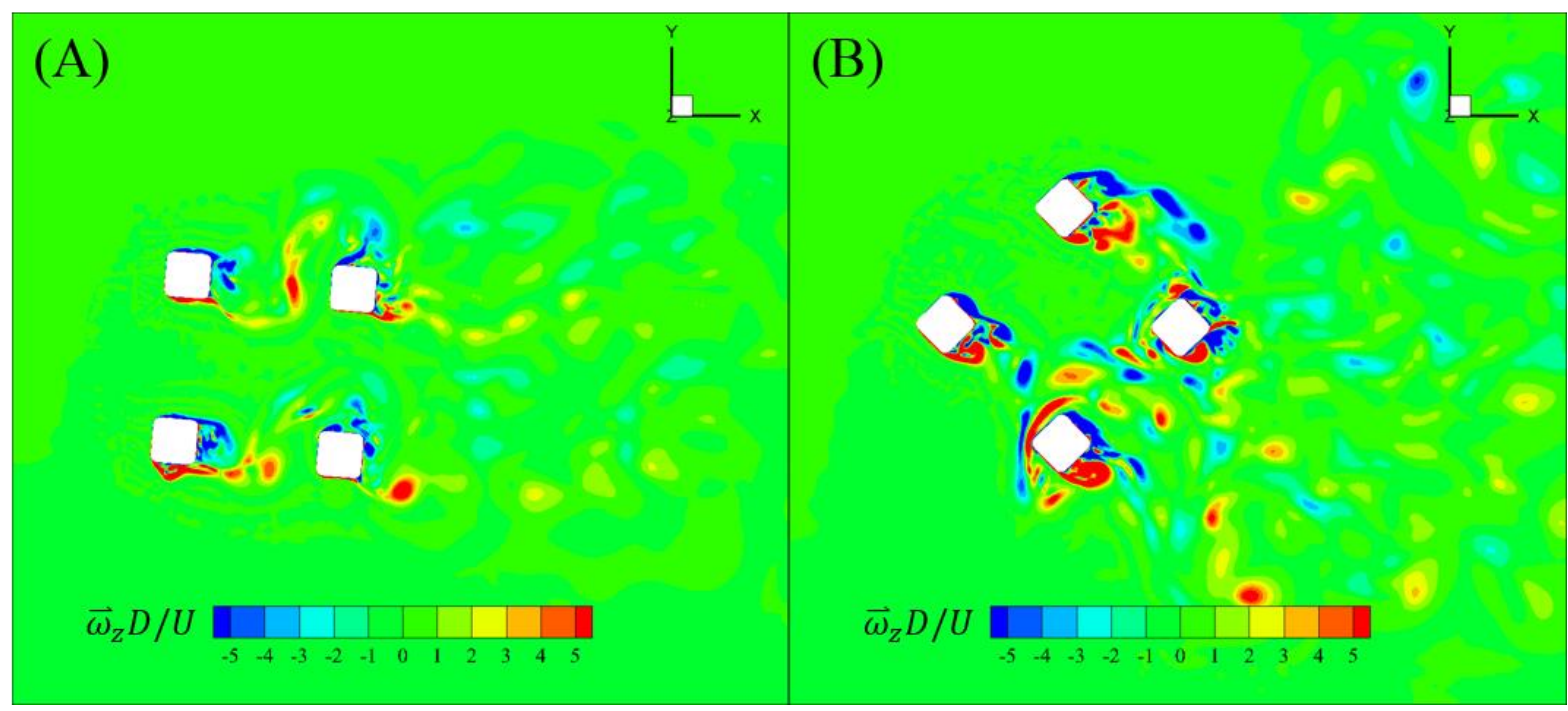

255 Fig. 6. Non-dimensional spanwise vorticity $\left(\vec{\omega}_{z} D / U\right)$ contours around the DDS at middle draft

256 showing the flow fields when "lock-in" has occurred (A: $U r=6.4,0$ degree incidence. B: $U r=6.6,45$

257 degree incidence).

\subsection{Stationary model results and observations}

In this section, a numerical study of the flow over a stationary deep-draft semi-submersible model

260 with Reynolds numbers varying from $3.7 \times 10^{4}$ to $6.0 \times 10^{4}$ was carried out in order to investigate the 


\begin{tabular}{cccc}
\hline $\boldsymbol{R} \boldsymbol{e}$ & $\overline{\boldsymbol{C}}_{\boldsymbol{D}}$ & $\boldsymbol{C}_{\text {Lrms }}$ & $\boldsymbol{S t}$ \\
\hline $3.7 \times 10^{4}$ & 1.068 & 0.070 & 0.140 \\
$4.3 \times 10^{4}$ & 1.046 & 0.069 & 0.138 \\
$5.2 \times 10^{4}$ & 1.044 & 0.066 & 0.143 \\
$6.0 \times 10^{4}$ & 1.053 & 0.080 & 0.142 \\
\hline
\end{tabular}

These numerical results are validated by the force measurements obtained from the corresponding experimental data. The experiments were conducted in a circulating water channel. The circulating water channel is vertically oriented with an $8.0 \mathrm{~m}$ length, $3.0 \mathrm{~m}$ width and $1.6 \mathrm{~m}$ depth measuring section. The range of the flow velocity is $0.1 \sim 3 \mathrm{~m} / \mathrm{s}$, and the minimum fluctuations of the current velocity speed is $0.01 \mathrm{~m} / \mathrm{s}$. The total fluid forces on the model I was measured by a three-component force transducer.

Comparisons between the results from the numerical simulations and the experimental measurements are shown in Fig. 7, Fig. 8 and Fig. 9. The numerical predictions for the mean drag coefficient $\left(\bar{C}_{D}\right)$, the root mean square lift force coefficient $\left(C_{\text {Lrms }}\right)$ and the Strouhal number $(S t)$ all show good agreements when compared with the experimental data. The drag and lift force coefficients on the structures remain stable in both the numerical and experimental results. The numerically predicted 
281 Strouhal number $(S t)$ is around 0.14 which is similar to the results from the CFD study carried out by 282 Lee et al. (2014).

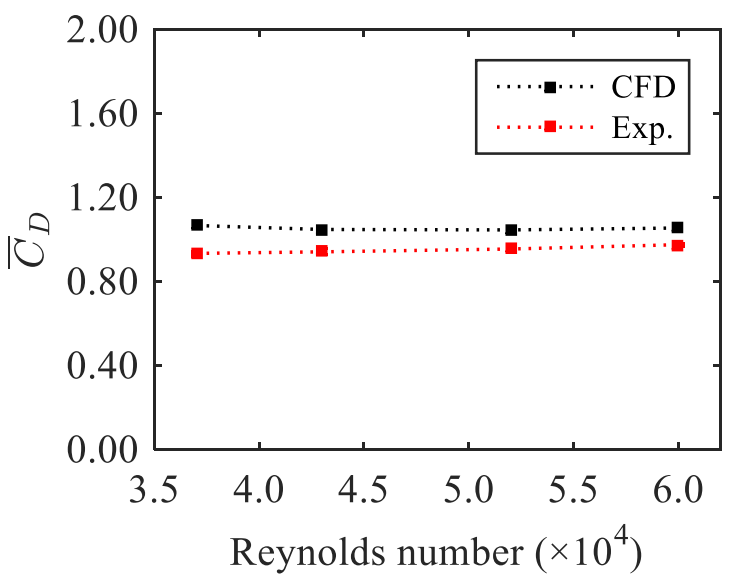

284 Fig. 7. Mean drag coefficient $\left(\bar{C}_{D}\right)$ from the numerical and experimental results for the stationary model.

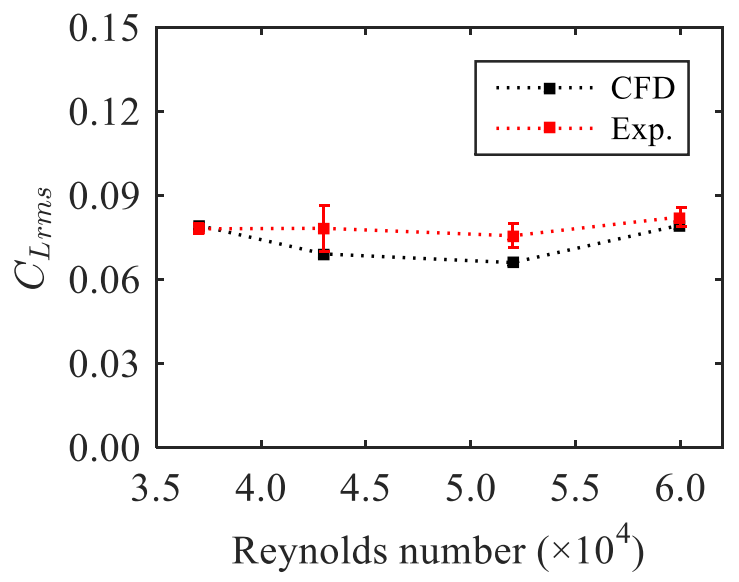

287 Fig. 8. Root mean square lift coefficient $\left(C_{\text {Lrms }}\right)$ from the numerical and experimental results for the 288 stationary model. 


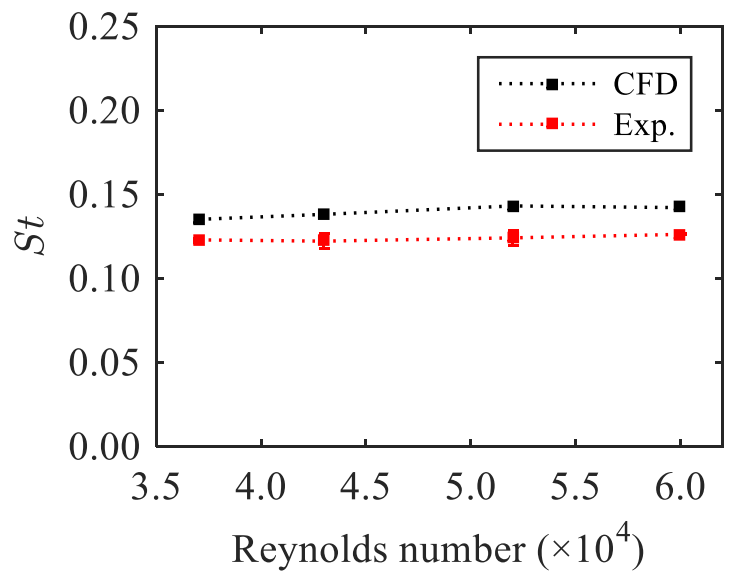

290 Fig. 9. Strouhal number (St) from the numerical and experimental results for the stationary model.

\subsubsection{Drag and lift forces on each member of the DDS}

292 In order to improve the understanding of interactions between vortex shedding processes due to each

293 structure member of the DDS, the drag and lift force coefficients on each member of the DDS are

294 calculated and presented in Fig. 10, Fig. 11 and Fig. 12. The mean drag coefficients on each member

295 remain stable within the current Reynolds number range similar to the trend of the overall mean drag

296 coefficients on the DDS as discussed in the above section. Respectively, the upstream column

297 (Column 1) experiences a larger mean drag coefficient $\left(\bar{C}_{D}\right)$ than the downstream one (Column 3 ).

298 The portside and starboard side columns (Column 2 and 4) are symmetrically expose to the flow and

299 experience a slightly larger mean drag coefficient $\left(\bar{C}_{D}\right)$ than the upstream column, and the pontoon

300 shows the same trend as the side columns do. It is noted that the downstream column and the two side

301 columns are subjected to higher fluctuating lift force coefficient than the upstream one with the

302 downstream column experiencing the largest fluctuating lift force coefficient among all parts of the

303 DDS. The root mean square lift force coefficients on the two side columns are slightly less than that

304 on the downstream one, but still much larger than that on the upstream column. These results show

305 the influence of unsteady vortices and their interactions on the structure members in the downstream.

306 Similar observation was also reported by Lam et al. (2003b). 


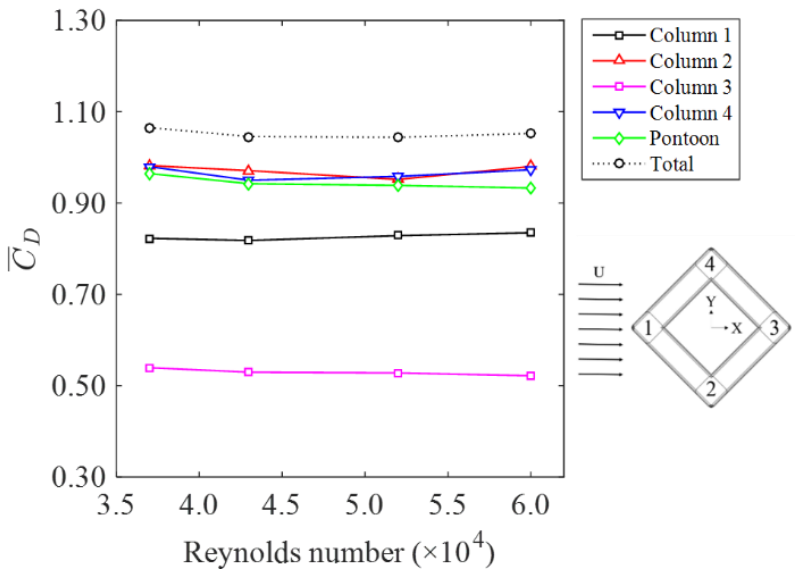

308 Fig. 10. Mean drag coefficients $\left(\bar{C}_{D}\right)$ on each member of the stationary DDS.

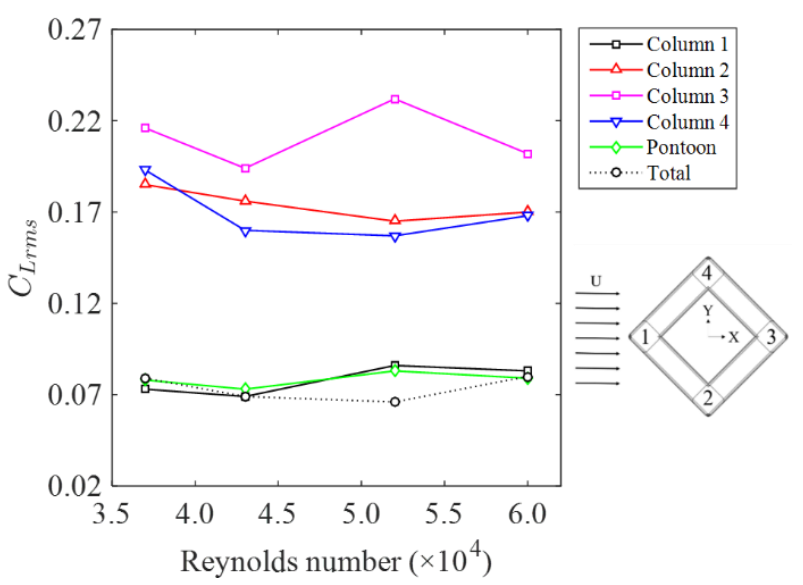

310 Fig. 11. Root mean square lift coefficients $\left(C_{\text {Lrms }}\right)$ on each member of the stationary DDS.

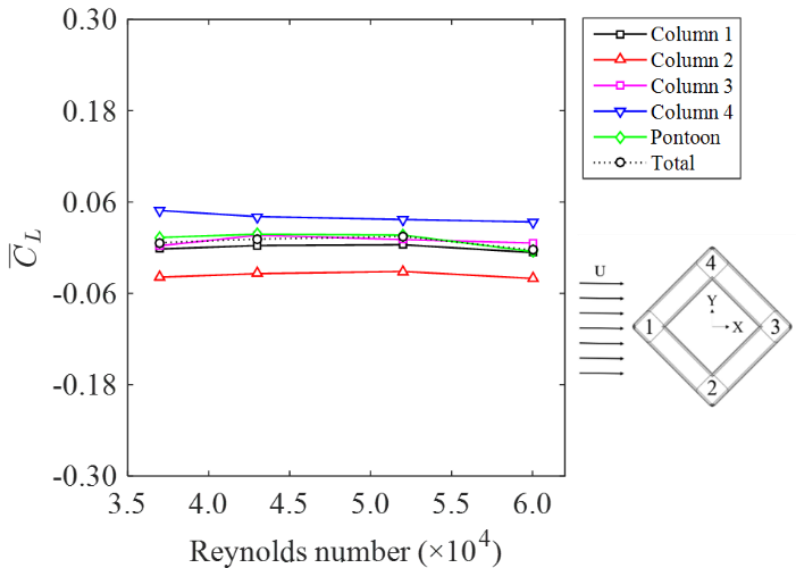


312 Fig. 12. Mean lift coefficient $\left(\bar{C}_{L}\right)$ on each member of the stationary DDS.

\subsubsection{Flow patterns and the lift force time history}

314 With the aim to reveal the force dynamic behaviours on the structure, the time histories of the lift

315 force coefficients corresponding with the flow patterns at $R e=4.3 \times 10^{4}$ are presented in Fig. 13, Fig.

31614 and Fig. 15.

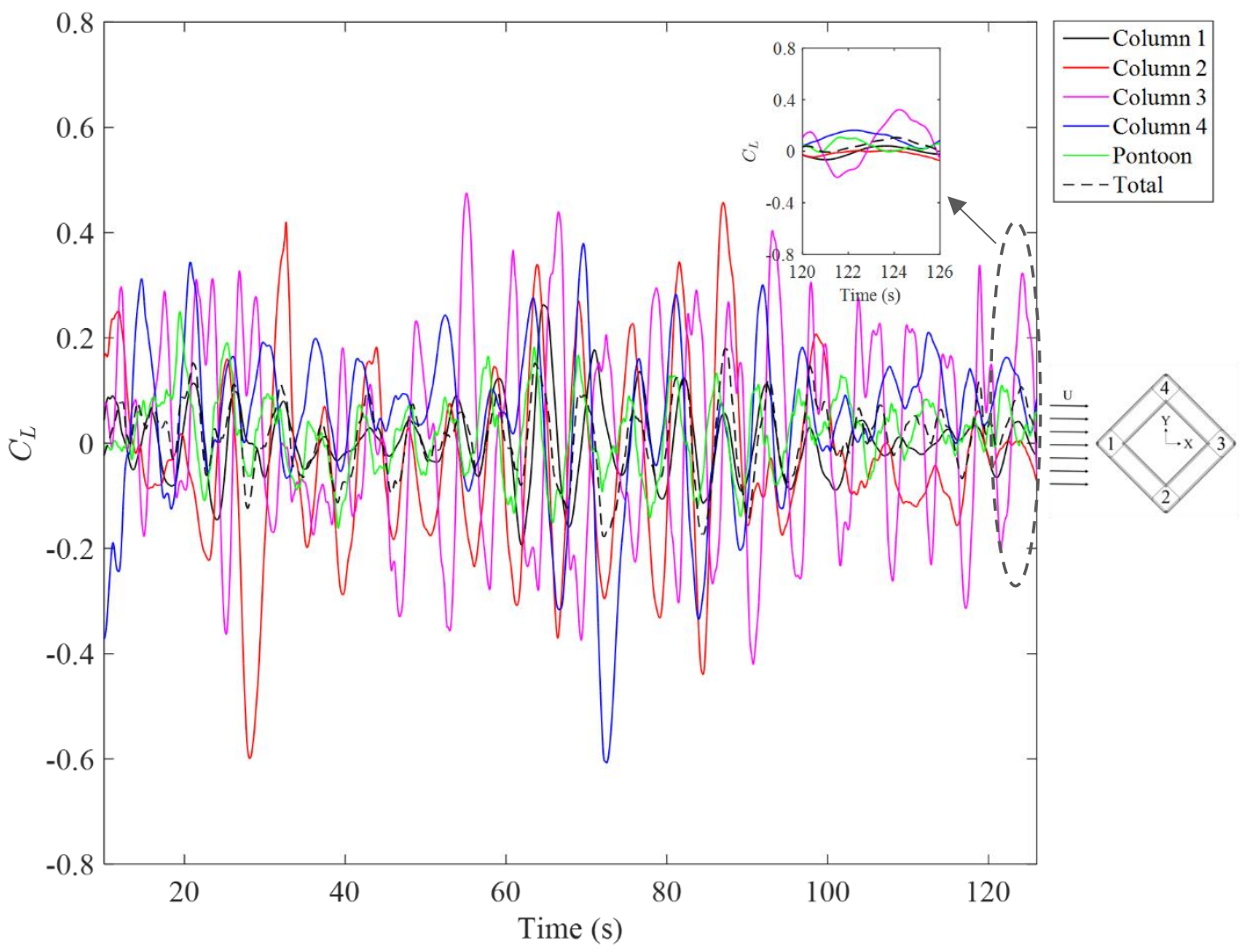

318 Fig. 13. Lift force coefficient time history on different members of the DDS at $R e=4.3 \times 10^{4}$, 319 including locally zoomed in the last $6 s$.

320 As can be seen in Fig. 13, the time history of the lift force coefficient on column 3 shows a hysteresis phenomenon which indicates the lift force oscillating period on column 3 always delayed compared with other structure members. The peak values of the lift coefficient on column 3 are almost 
corresponded to the bottom values of other structure members, and vice versa. From the pressure contours (Fig. 14), it can be clearly observed that there is a relatively stationary high pressure zone in front of column 1, 2 and 4. However, the high pressure zone in front of the downstream column 3 keeps changing along with the vortex shedding processes. The fluctuations of the pressure in front of column are primarily induced by the impingement of the upstream generated vortices, and these fluctuations of the pressures cause the downstream column 3 to have higher $C_{\text {Lrms }}$ and lower $\bar{C}_{D}$ values compared with other three upstream columns. On the other hand, the pressure fluctuations in front of column 3 are mainly resulted in the interaction between the vortices shed from the upstream column 1 and the shear layers separated from the downstream column 3, which can be clearly seen in
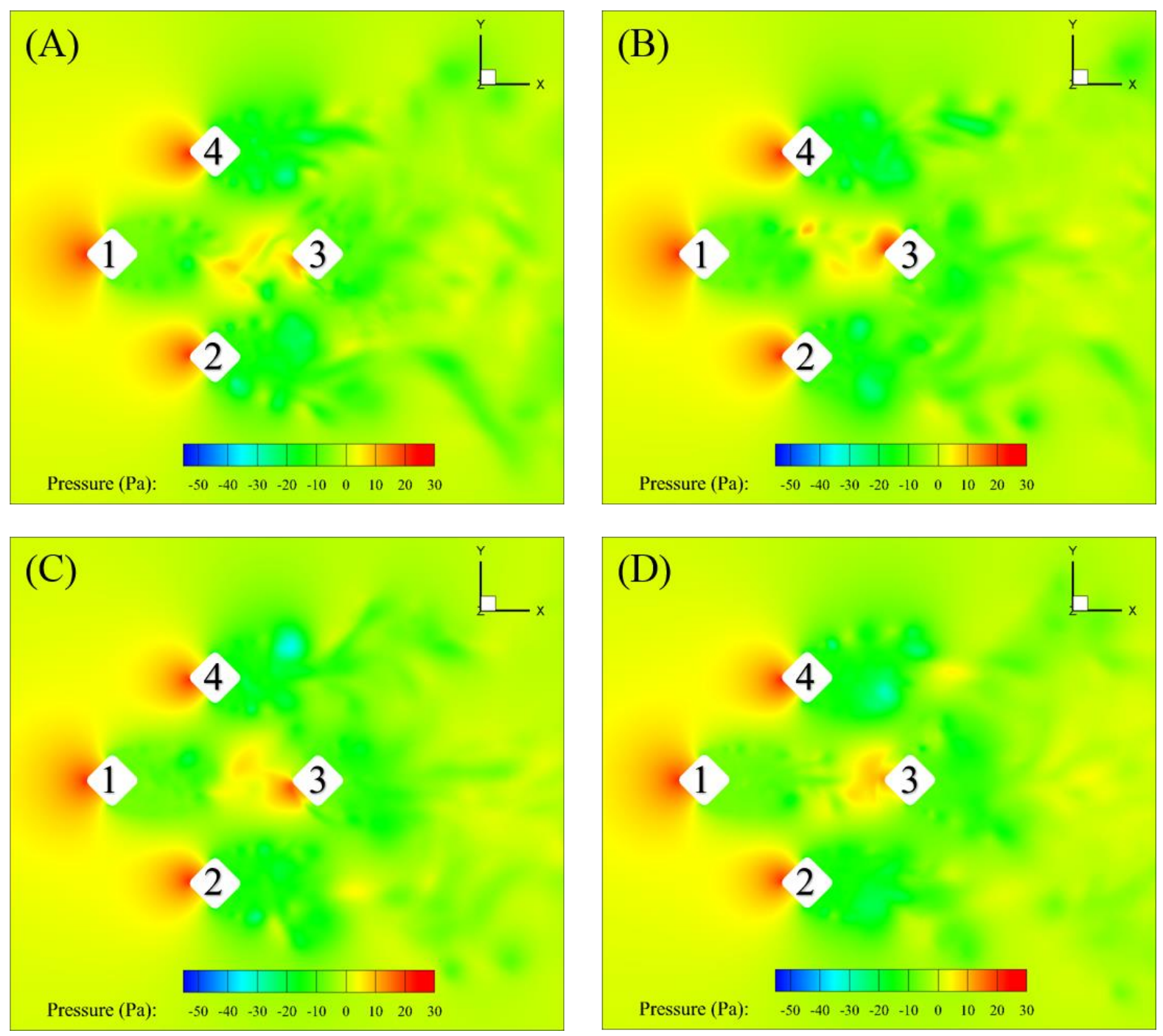

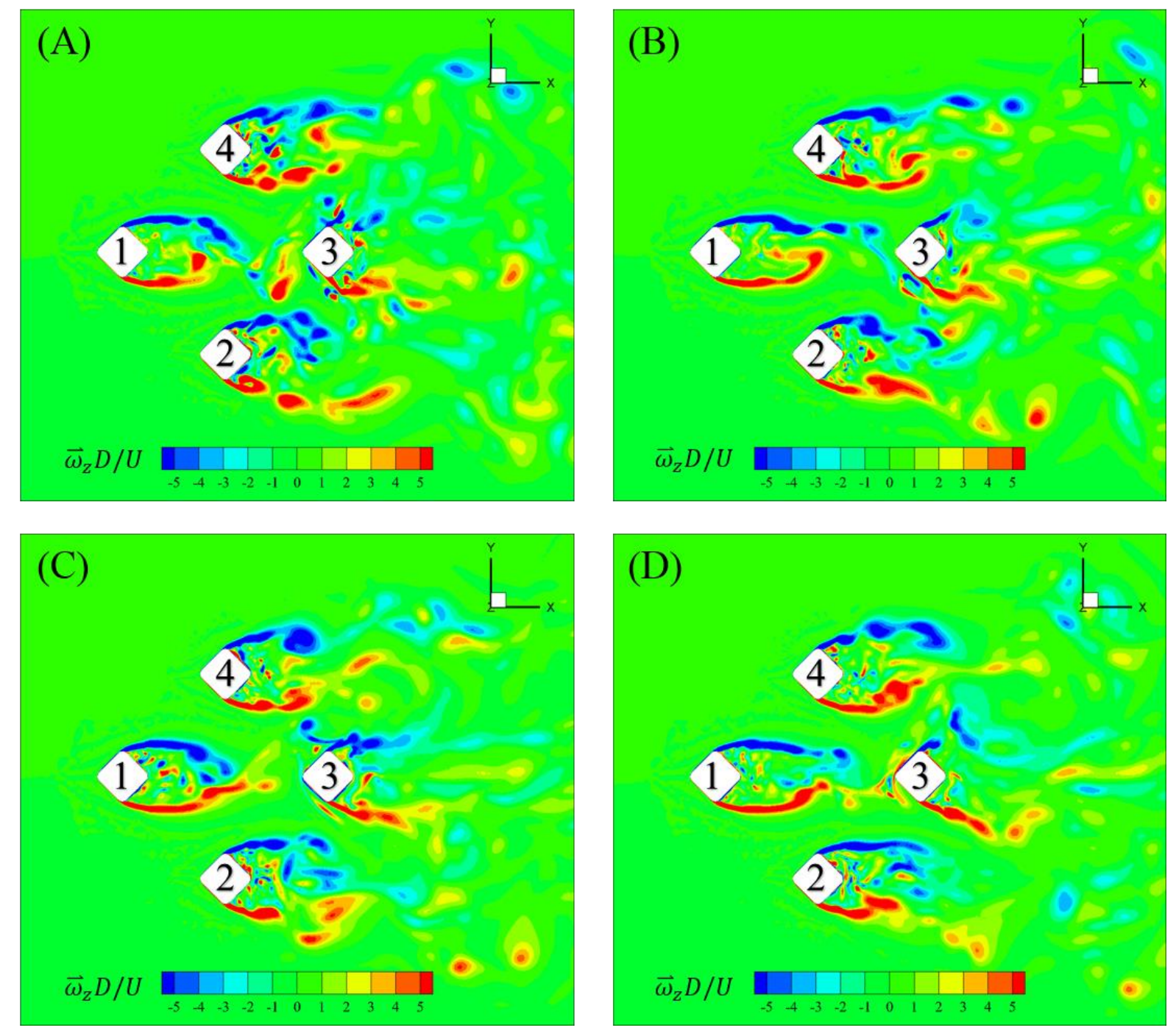

Fig. 15. A time series of non-dimensional spanwise vorticity $\left(\vec{\omega}_{z} D / U\right)$ contours around the DDS at the middle draft level showing the instantaneous flow fields around the DDS at $R e=4.3 \times 10^{4}$ corresponding to the lift force coefficient time history (A: 120.6s; B: 122.4s; C: 124.2s; D: 126.0s).

342 Fig. 15 also shows that the vortex shedding patterns due to each column are very different. It is seen 343 that very slim vortices are shed from the corners of column 1 . However, the vortices shed from the 344 two side columns, i.e., column 2 and column 4 appear to be not as slim as the ones of column 1, and 
even shorter vortices shed from the corners of downstream column 3 are clearly visible. Moreover, column 2 and column 4 shed the large vortices symmetrically, where column 2 shed the vortices on its portside corner and the column 4 shed on its starboard side corner. This symmetrical vortex shedding pattern contributes to the symmetrical values of $\bar{C}_{L}$ for column 2 and column 4 as shown in Fig. 12.

After studying the vortex shedding interactions with the columns for the flow over a stationary structure, a VIM investigation was carried out, in order to reveal the cause of VIM by comparing the forces distributions and the flow patterns differences between the flow over the motion-coupled structure cases with the stationary structure cases.

In this section, the numerical simulations of the flow over a three degree of freedom deep-draft semisubmersible model with different Reynolds numbers from $3.6 \times 10^{4}$ to $1.1 \times 10^{5}$ are carried out to investigate the overall hydrodynamics of the structure. Results for all cases are obtained by averaging after more than ten vortex shedding cycles. Although the sample size is relatively small, the reliability and sensitivity of the relatively small data set on the results have been discussed by Zhang et al. (2014). These numerical predictions are subsequently validated by the motion and force measurements obtained from the corresponding experiments undertaken in a towing tank. The characteristics of vortex shedding processes and their interactions due to multiple cylindrical columns are also discussed.

\subsubsection{Experimental test}

Table 5. Natural periods of the motions in calm water.

\begin{tabular}{llll}
\hline Incidence $\left(^{\circ}\right)$ & $\begin{array}{l}\text { Natural period of } \\
\text { transverse motion, } \\
\boldsymbol{T}_{\text {otransverse }}(\mathbf{s})\end{array}$ & $\begin{array}{l}\text { Natural period of in- } \\
\text { line motion, } \\
\boldsymbol{T}_{\text {0in-line }}(\mathbf{s})\end{array}$ & $\begin{array}{l}\text { Natural period of } \\
\text { yaw motion, } \\
\boldsymbol{T}_{\text {oyaw }}(\mathbf{s})\end{array}$ \\
\hline $\mathbf{4 5}^{\circ}$ & 20.1 & 19.2 & 18.3 \\
\hline
\end{tabular}


365 In order to validate the numerical model, a series of experiments were performed in a towing tank

366 with a dimension of $130 \times 6 \times 3 \mathrm{~m}$ (length $\times$ width $\times$ depth). The model II described in Table 1 was

367 tested under a reduced velocity $(U r)$ ranging from 3.4 to 14.1. A minimum of ten oscillation cycles

368 were allowed to occur in order to reach the quasi-steady state of the VIM phenomenon. Only three

369 degrees of freedom (namely transverse, in-line and yaw) were allowed in the experiments. The motion

370 time histories were recorded and the forces on mooring lines were measured to obtain the

371 hydrodynamic loads on the model. Table 5 lists the natural periods of the motions in calm water

372 obtained from the decay tests.

\subsubsection{Motion characteristics}

374 The non-dimensional characteristic amplitudes are introduced in this section to describe and present

375 the VIM motion characteristics. The non-dimensional characteristic amplitudes are defined as:

376

$A_{x} / L=\sqrt{2} \times \sigma\left(\frac{x(t)}{L}\right)$,

$377 \quad A_{y} / L=\sqrt{2} \times \sigma\left(\frac{y(t)}{L}\right)$,

$378 Y Y_{\text {nom }}=\sqrt{2} \times \sigma(\operatorname{yaw}(t))$,

379 where $L$ is the column width, $\sigma$ is the standard deviation obtained from the time series, $x(t), y(t)$ and

$380 y a w(t)$ represent the in-line, transverse and yaw motions time series respectively. 


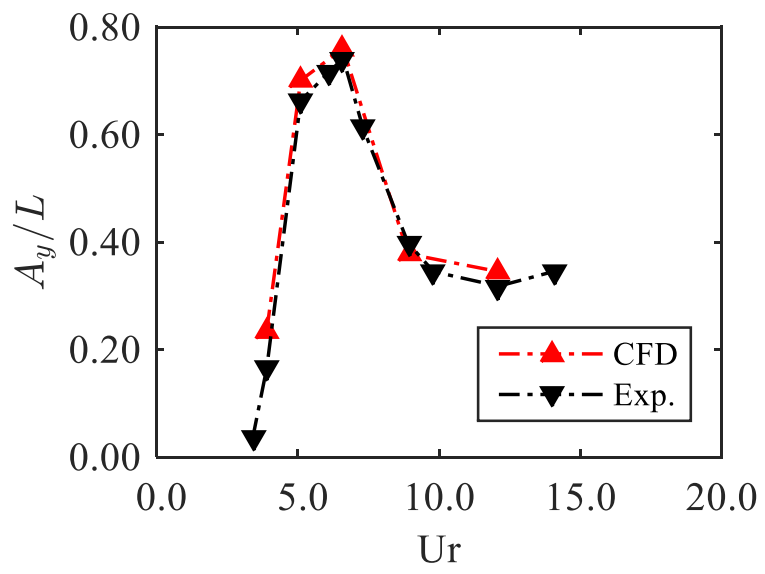

382 Fig. 16. Non-dimensional transverse characteristic amplitudes $\left(A_{y} / L\right)$, the $U r$ is defined based on the 383 natural period of the transverse motion.

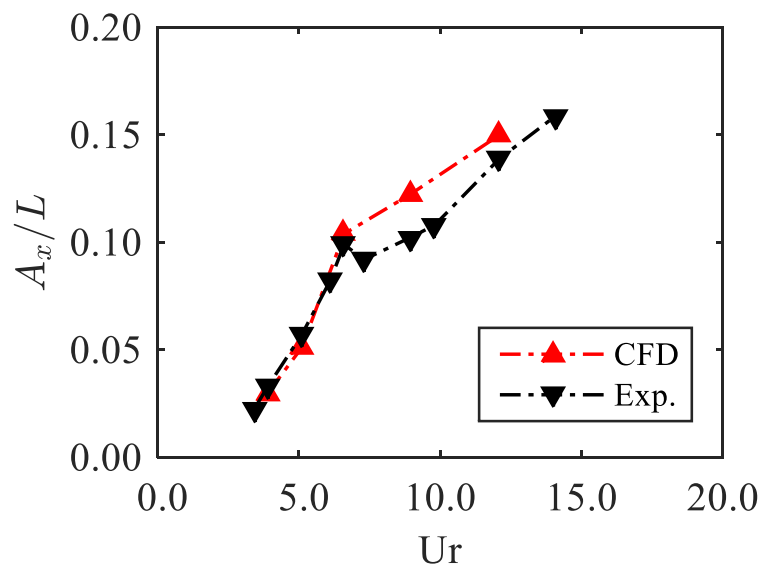

385 Fig. 17. Non-dimensional in-line characteristic amplitudes $\left(A_{x} / L\right)$, the $U r$ is defined based on the 386 natural period of the transverse motion. 


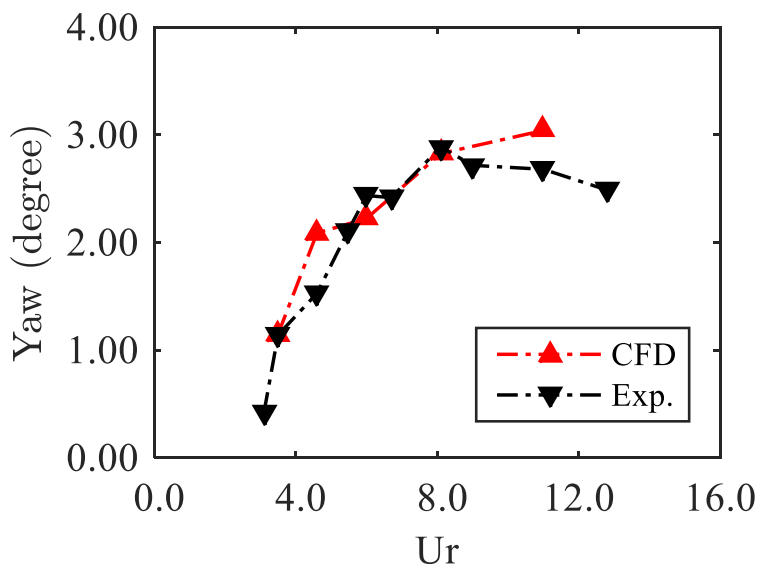

Fig. 18. Non-dimensional yaw characteristic amplitudes, the $U r$ is defined based on the natural period of the yaw motion.

Fig. 16, Fig. 17 and Fig. 18 present the non-dimensional transverse, in-line and yaw motion amplitudes obtained from the numerical simulations and the experimental measurements. In each case, the numerical predictions show a good agreement with the experimental results. However, at low $U r$ values, the numerical simulation predicts a slightly larger transverse response than the experimental data. An analysis of the error will be given in the following 3.3.3. Force analysis section together with the added mass analysis. From the non-dimensional transverse characteristic amplitude in Fig. 16, the "lock-in" phenomenon can be clearly seen occurring in a reduced velocity range from 5 to 9. The transverse motion increased rapidly from the "pre lock-in" region to the "lock-in" region, and then sharply declines from the "lock-in" region to the following "post lock-in" region. The peak point for the transverse motion is at $U r=6.6$.

\subsubsection{Force analysis}

401 In order to study the fluctuation forces responsible for VIM, the lift and drag forces and the related 402 coefficients are further analysed. Fig. 19 and Fig. 20 show the mean drag force coefficient $\left(\bar{C}_{D}\right)$ and 403 the root mean square lift force coefficient $\left(C_{L r m s}\right)$ respectively as a function of the reduced velocity $404(U r)$ for both numerical and experimental results. The numerical method predicts well compared with 

and the experimental data at low reduced velocity levels for the root mean square lift force coefficient, similar to the trend observed in the non-dimensional transverse characteristic amplitudes in Fig. 16.

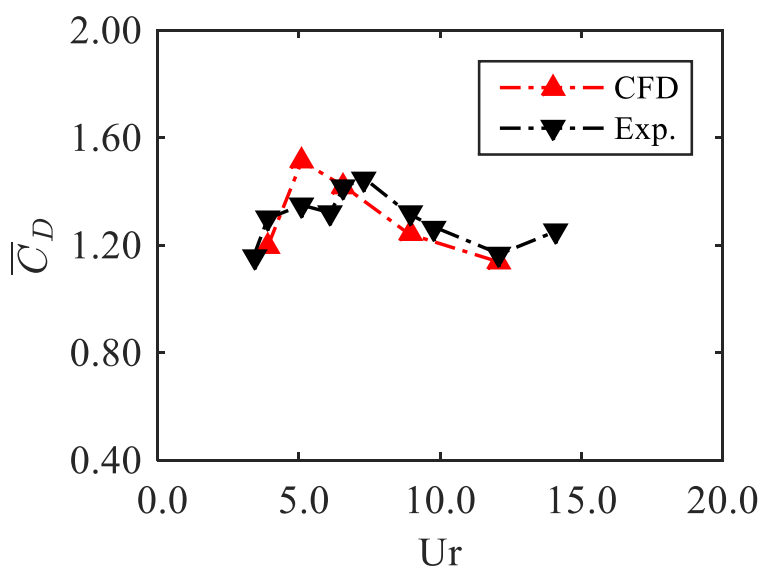

409 Fig. 19. Mean drag coefficient $\left(\bar{C}_{D}\right)$ from the numerical and experimental results on the VIM model, 410 the $U r$ is defined based on the natural period of the transverse motion.

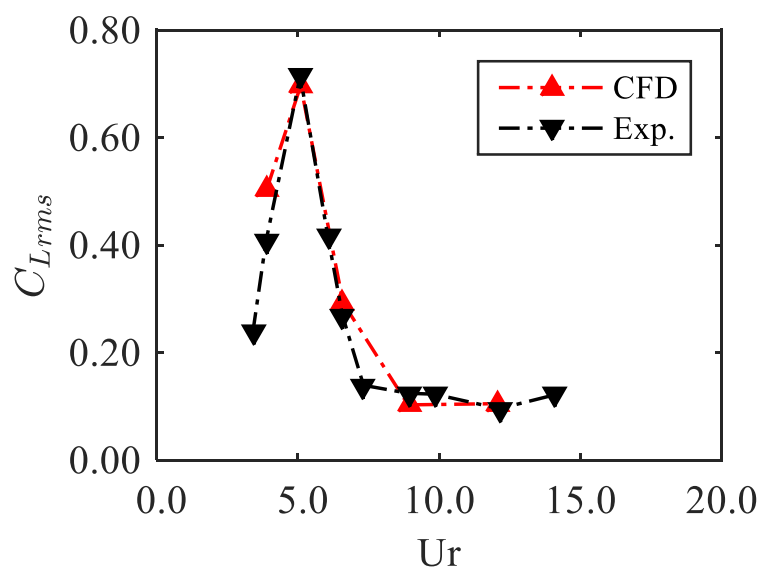

411

412 Fig. 20. Root mean square lift coefficient $\left(C_{\text {Lrms }}\right)$ from the numerical and experimental results on the

413 VIM model, the $U r$ is defined based on the natural period of the transverse motion.

414 To further examine the differences, a virtual variable so-called added mass coefficient $\left(C_{a}\right)$ has been 415 introduced to compare the differences between the numerical predictions and the experimental 
416 measurements. Similar to the discussions made by Sarpkaya (2004) in their vortex-induced vibrations

417 study, Zhang et al. (2014) introduced this variable into a Spar VIM investigation. In their work, the

418 added mass coefficient $\left(C_{a}\right)$ is estimated by the equations proposed by Vikestad et al. (2000) as

419 follows:

$420 \quad C_{a}=\frac{m_{a}}{\rho \Delta}$,

$421 \quad C_{a}=-\frac{2}{n T \rho \Delta(\sqrt{2} r m s(\dot{y}))^{2}} \int_{t}^{t+n T} F_{y} \ddot{y} d t$

where $n$ is an integer number of oscillation periods, $n T$ is the time length, $\rho$ is the fresh water density,

$\Delta$ is the displacement of the structure, $F_{y}$ is the cross-flow component of the total hydrodynamic force on the structure and $y$ is the transverse displacement of the motion.

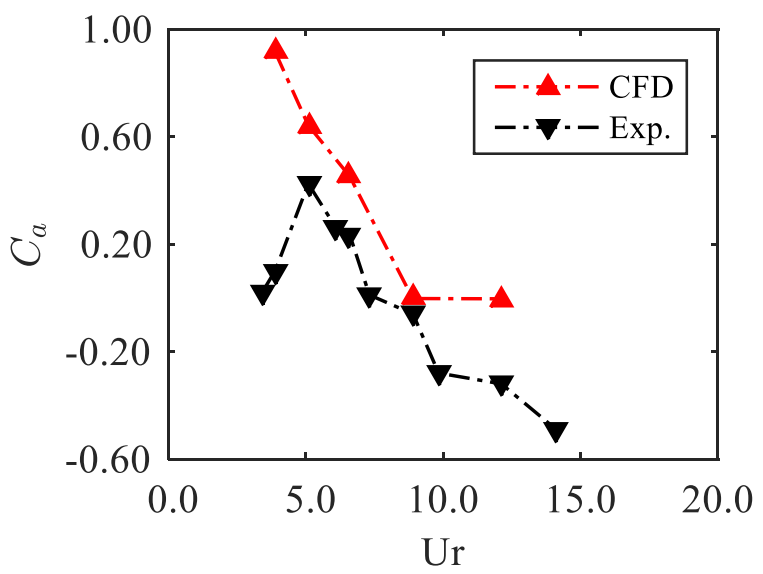

Fig. 21. Added mass coefficient $\left(C_{a}\right)$ of the VIM model from the numerical predictions and the experiments, the $U r$ is defined based on the natural period of the transverse motion.

Fig. 21 shows the comparison of the added mass coefficients $\left(C_{a}\right)$ obtained from the numerical calculations and the experiments. The numerical prediction shows a decreasing trend similar to that reported in an earlier study by Zhang et al. (2014). This trend is also the same as the results from the studies conducted by Sarpkaya (2004). However, the added mass coefficient $\left(C_{a}\right)$ obtained from the 
experimental measurements at low reduced velocity range are significantly different to those from the present numerical predictions. A distinct feature shown in Fig. 21 is that the added mass coefficient from experiments is much smaller at very low $U r$ and trends to increase initially and then decrease rapidly with the increases of the reduced velocity. The apparent discrepancy between the numerical and experimental results at low reduced velocities is likely to be caused by the experimental data. There are a few possibilities that could cause the error. Firstly, the towing speed during the experiment is extremely low for an equivalent low reduced velocity (for example, $0.073 \mathrm{~m} / \mathrm{s}$ for reduced velocity at $U r=3.4$ ), and the whole system mechanical friction may affect the experimental measurements at such a low towing speed; secondly, the influence of the mooring line settings may also affect the experimental measurements, because the theoretically linear springs set in numerical simulations are ideal springs and the mooring lines in the experimental set-up may not be arranged as symmetrically as in the numerical simulations. Due to these factors, the numerical results may be more reliable and accurate than the experimental data in the low reduced velocity range.

Similar to the motion observation, the "lock-in" phenomenon can also be seen in Fig. 19 and Fig. 20. However, it is noted that the "lock-in" phenomenon in the force domain is seen to occur slightly earlier than in the transverse motion domain, as also observed by Gonçalves et al. (2012) in their experiments. The peak point for the drag and lift force coefficients in the present study are at $U r=5.1$ while the peak point for the transverse motion is at $U r=6.6$.

Both the transverse motion time histories and the lift force coefficient time histories are transferred from the time domain to the frequency domain by using the Fast Fourier transform (FFT) in order to study the "lock-in" phenomenon. The frequency domain results are shown in Fig. 22 to Fig. 26, inclusive the "pre lock-in", "lock-in" and "post lock-in" regions. The transverse motion frequency and vortex shedding frequency are both close to the transverse natural frequency in still water at the "pre lock-in" and "lock-in" regions. The oscillation and vortex shedding frequency are shown increasing with the increase in reduced velocity. When the "post lock-in" started, the oscillation frequency and vortex shedding frequency started to be further away from the transverse motion natural frequency as 
can be seen in Fig. 25. For the highest reduced velocity case at $U r=12.1$, Fig. 26 shows multiple peak frequencies appearing in the frequency domain.

460

461

462

463

464

465

466

467

468

469

470

As can be seen in Fig. 22 to Fig. 25, the agreement between the numerical predictions and the experimental measurements for both transverse motions and the lift force coefficients are reasonably well. It is seen that in Fig. 26, however, at " $U r=12.1$ ", the agreement is less well especially the magnitudes of motion and force coefficient though the dominant frequencies were still predicted accurately. It is noted that, at such a high reduced velocity $(U r=12.1)$ far beyond the "lock-in" region (approx. $U r=6.6$ ), the magnitudes of the transverse motion and lift force coefficient are much smaller, thus, the relatively larger discrepancies appeared in Fig. 26.

Compared to Fig. 24 with $U r=6.6$, Fig. 23 shows that the oscillation frequency and vortex shedding frequency are closer to the transverse natural frequency at $U r=5.1$, where the values of the peak drag and lift force coefficients appear. Furthermore, the added mass may also contribute to the earlier peak drag and lift force occurrence. Since the added mass keeps decreasing with the reduced velocity increasing, the force domain and the motion domain may have a hysteresis phenomenon which requires further studies.
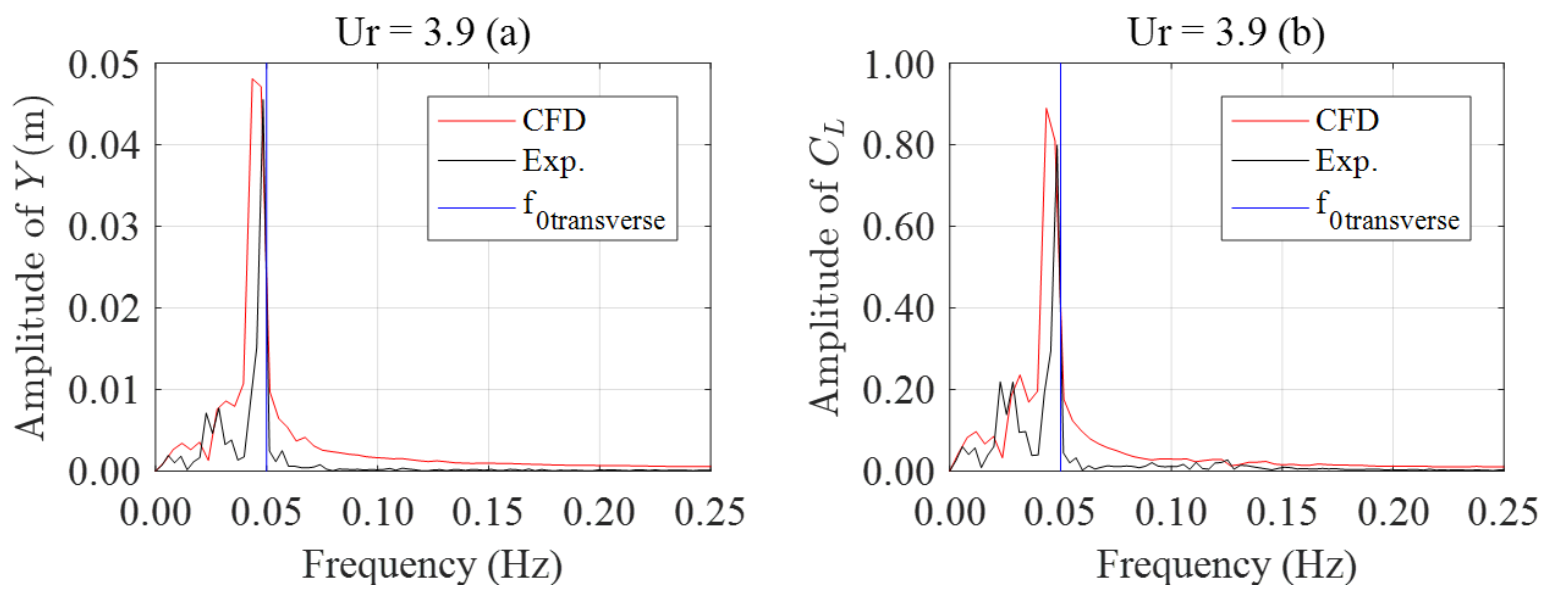

Fig. 22. FFT of the transverse motions and the lift force coefficients at $U r=3.9$, (a) transverse motion; (b) lift force coefficient. 

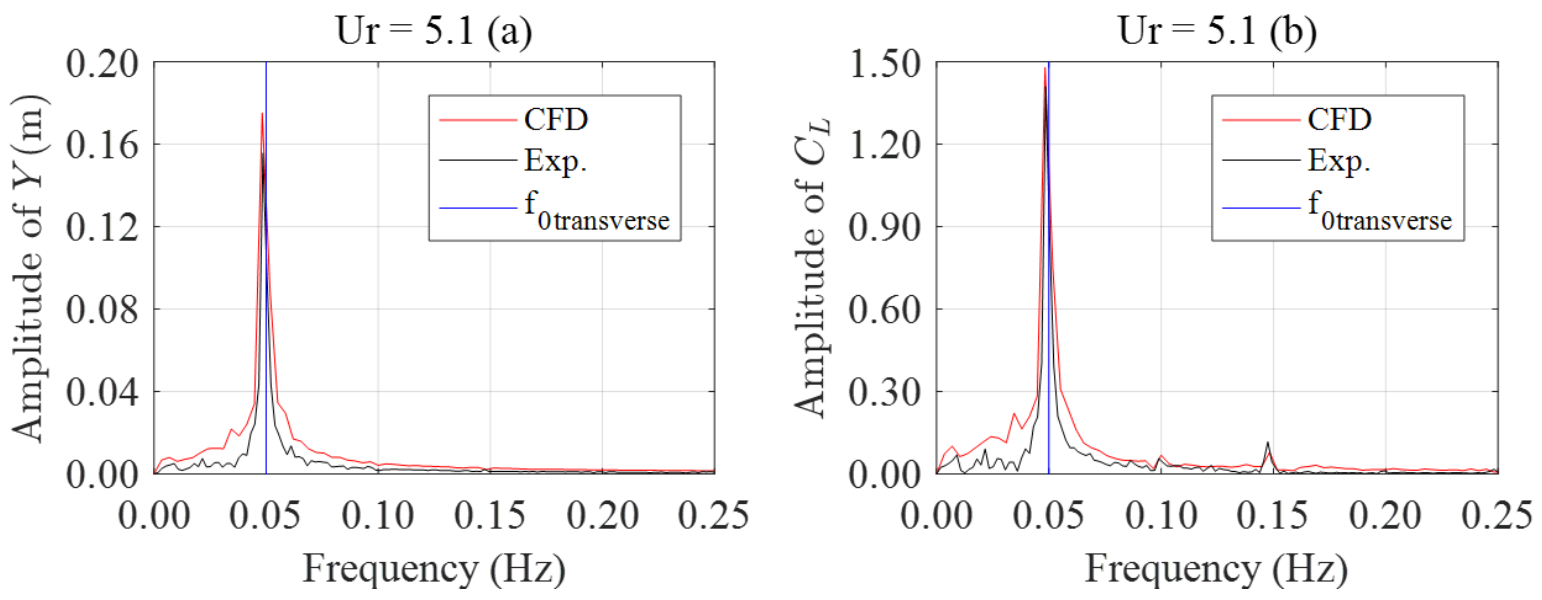

477 Fig. 23. FFT of the transverse motions and the lift force coefficients at $U r=5.1$, (a) transverse 478 motion; (b) lift force coefficient.
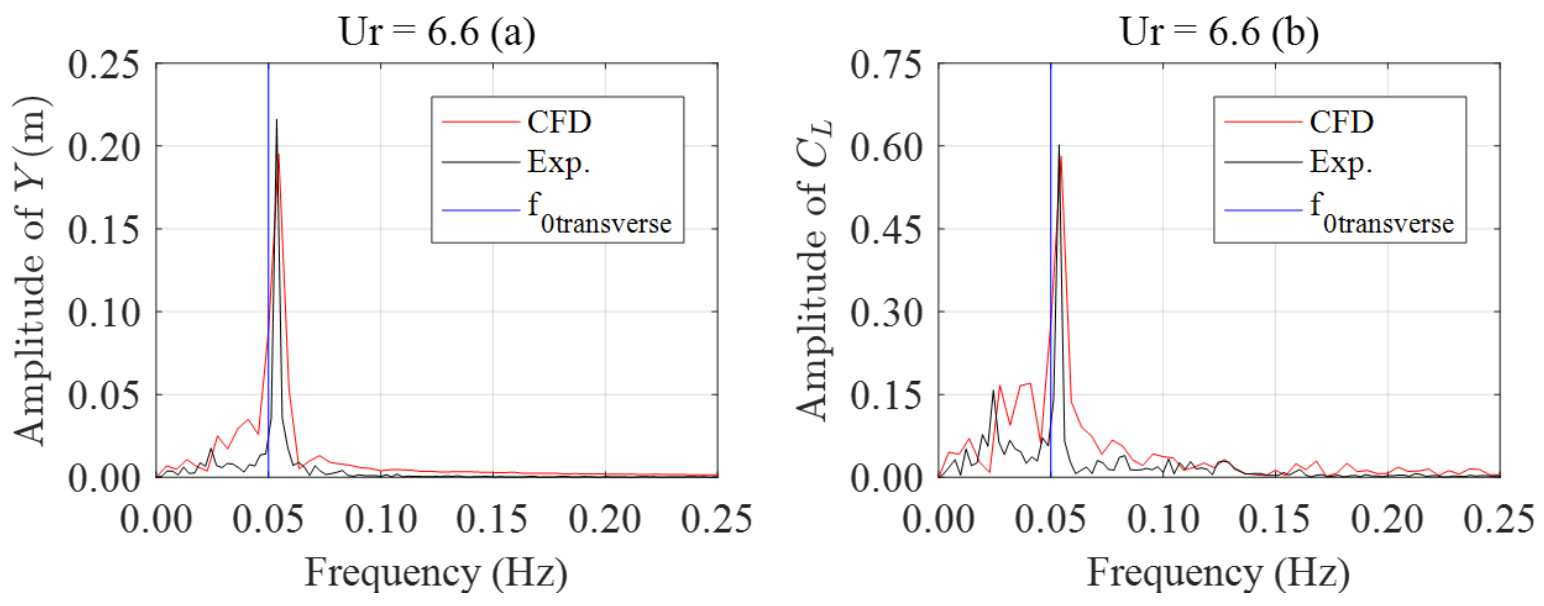

480 Fig. 24. FFT of the transverse motions and the lift force coefficients at $U r=6.6$, (a) transverse motion; (b) lift force coefficient. 

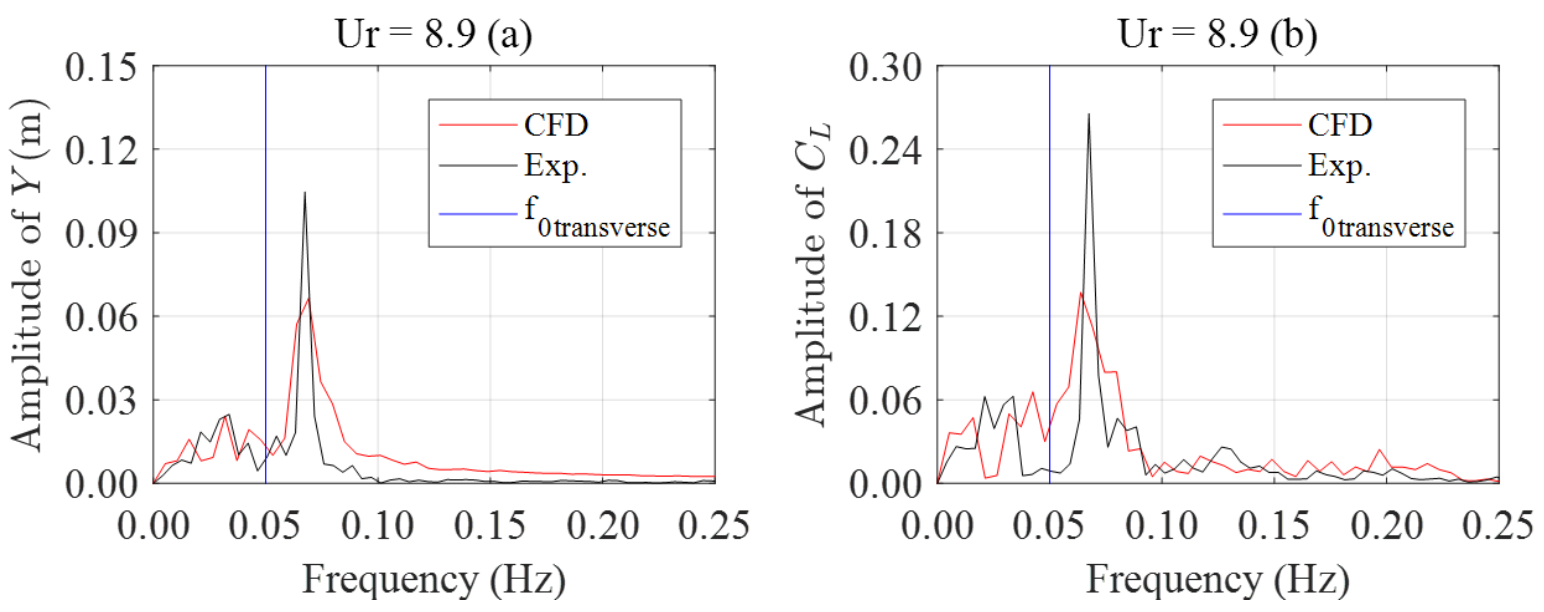

483 Fig. 25. FFT of the transverse motions and the lift force coefficients at $U r=8.9$, (a) transverse 484 motion; (b) lift force coefficient.
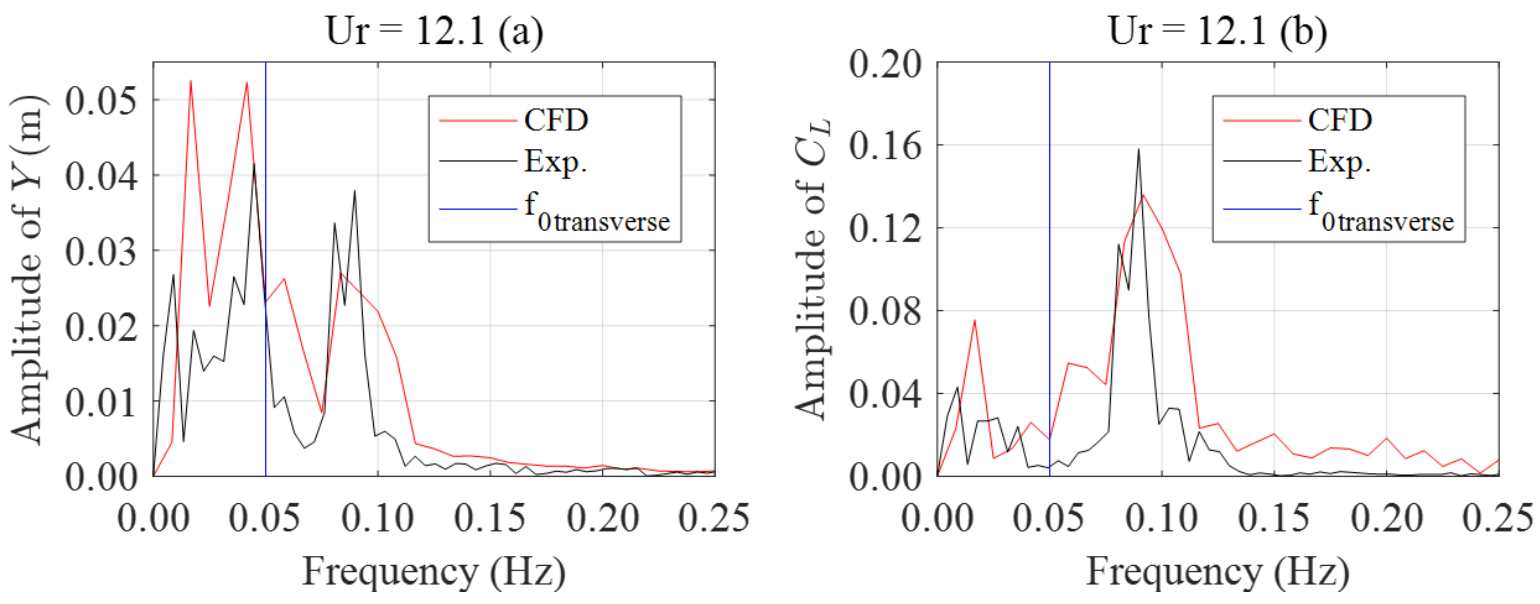

486 Fig. 26. FFT of the transverse motions and the lift force coefficients at $U r=12.1$, (a) transverse 487 motion; (b) lift force coefficient.

488 To examine the complex fluid mechanisms on the structure and the corresponding motion driven parts

489 of the structure, the drag and lift force coefficients on different structure members of the DDS are

490 further calculated and analysed.

491 Unlike the stationary model, the drag and lift force coefficients are changed when the reduced velocity increases (Fig. 27 and Fig. 28). The mean drag coefficient $\left(\bar{C}_{D}\right)$ on the upstream column 
493 (Column 1), the portside column (Column 2) and the starboard side column (Column 4) are excited by

494 the "lock-in" phenomenon. The pontoons are less excited compared to the three aforementioned

495 columns. However, the drag force coefficient on the downstream column (Column 3) is decreasing

496 while coefficients for the other members experiencing increasing trends and only recovers when "post

497 lock-in" phase starts. The drag force coefficient on the downstream column is also much smaller than

498 that for other members of the structure. On the other hand, the lift force coefficient $\left(C_{\text {Lrms }}\right)$ on the

499 downstream column, the portside and starboard side columns, and on the pontoons, are all excited by

500 the "lock-in" phenomenon. At this time, the leading upstream column shows a different trend. The lift

501 force coefficient on the upstream column is seen to decrease while an increasing trend is observed for

502 the other components, and conversely starts to recover as the other components begin to decrease.

503 This is due to the wake region changing behind each of the columns. Further details will be discussed 504 in the 3.3.4. Flow pattern section.

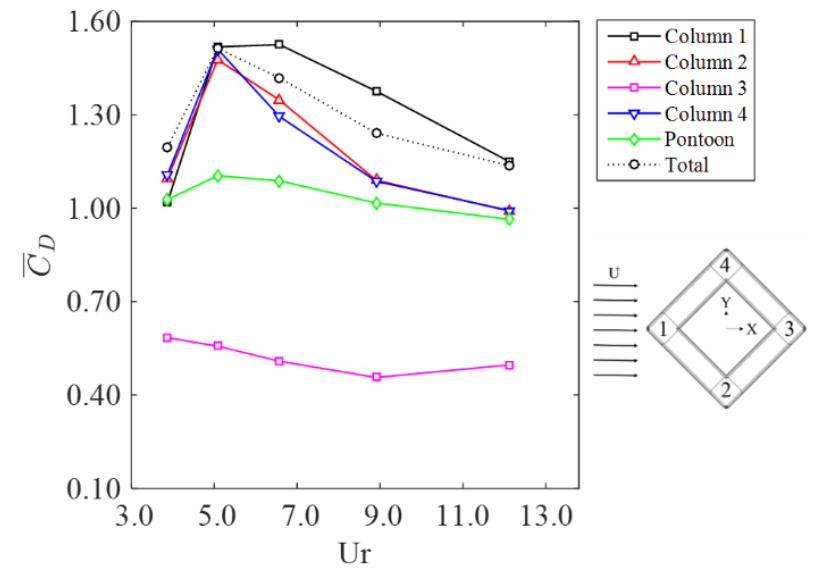

506 Fig. 27. Mean drag coefficients $\left(\bar{C}_{D}\right)$ on each member of the DDS from the VIM model. 


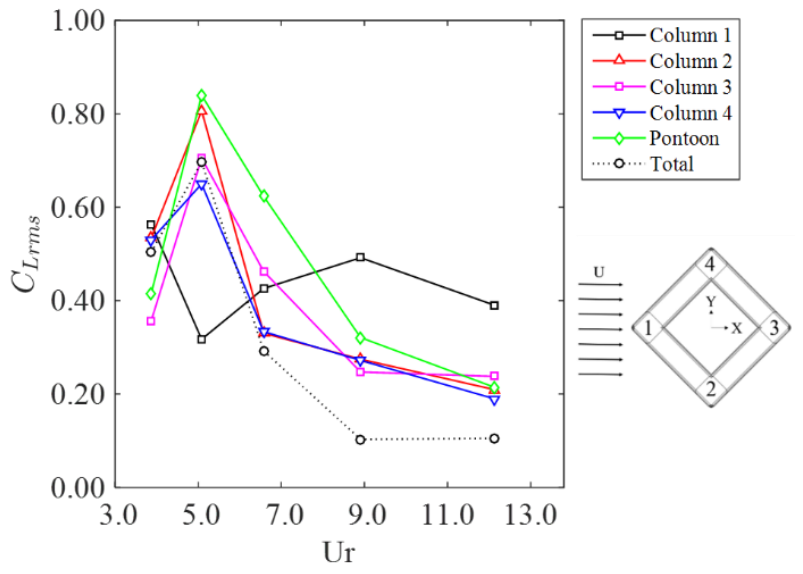

508 Fig. 28. Root mean square lift coefficients $\left(C_{L r m s}\right)$ on each member of the DDS from the VIM model.

509 The drag and lift forces on the structure are nearly symmetric except the lift force coefficient

510 distribution at $U r=5.1$. Due to the results being based on the motion-coupled simulations, the rigid

511 body motion also needs to be included in the analysis. With this aim, the work done by each member

512 of the structure during the stabilized VIM time is calculated and the results are presented in Fig. 29.

513 The work done is calculated using the following equations:

$514 \quad W=F \cdot S$,

515 where $F$ is the total drag and lift force of the structure and $S$ is the displacement of the structure 516 motion.

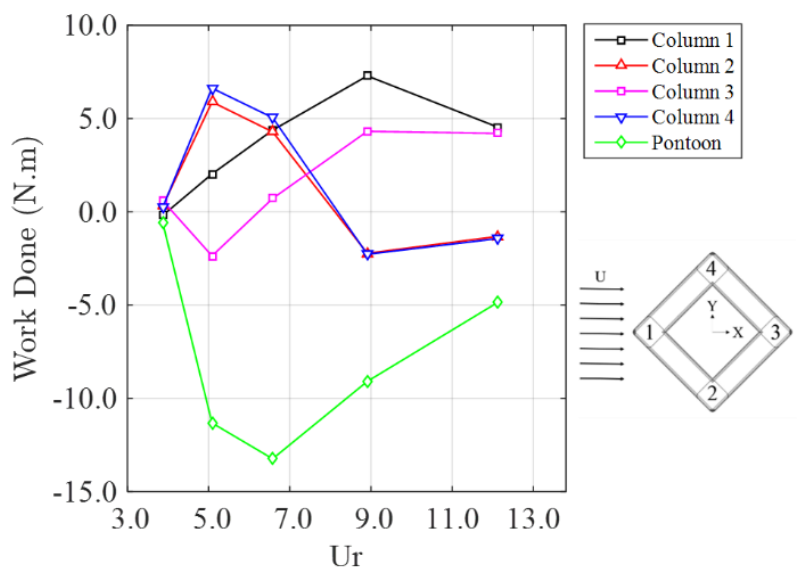


Fig. 29. Work done by each member of the DDS on VIM model.

519 In Fig. 29, the symmetrical characteristics can be clearly identified, and the following features can be 520 observed:

521

$\omega=\sqrt{\vec{\omega}_{x}^{2}+\vec{\omega}_{y}^{2}+\vec{\omega}_{z}^{2}}$ where, $\omega$ is the vorticity magnitude, $\vec{\omega}_{x}, \vec{\omega}_{y}$ and $\vec{\omega}_{z}$ are the $x, y$, and $z$ components of the vorticity, $D$ is

1) The pontoon reduces the VIM response throughout the reduced velocity range. Thus, adding on the pontoon into the overall structure is a good design for restraining VIM responses.

2) The three upstream columns excite VIM responses. Further, the portside and starboard side columns excite VIM responses in the "lock-in" region and trend to resist VIM responses in the "post lock-in" region.

3) The downstream column shows a different trend compared to the portside and starboard side columns; the work done by the downstream column drops initially and then recovers.

\subsubsection{Flow pattern}

In order to have a general visual appreciation of the vortex shedding patterns, the vorticity contours are plotted in Fig. 31 to Fig. 40. Two non-dimensional variables (non-dimensional vorticity and nondimensional spanwise vorticity) are used to describe the vorticity in the current study.

non-dimensional vorticity $=\omega D / U$, the projected length of the column and $U$ is the current speed. 
537 For convenience in describing the vortex development processes, four regions are defined around the

538 column, named as NW (Northwest), NE (Northeast), SW (Southwest) and SE (Southeast) (see Fig.

539 30). The vortices shed from each side of the column are denoted in chronological order of genesis

540 (e.g., A1, A2 ...) from the upper side of Column 1, see Table 6.

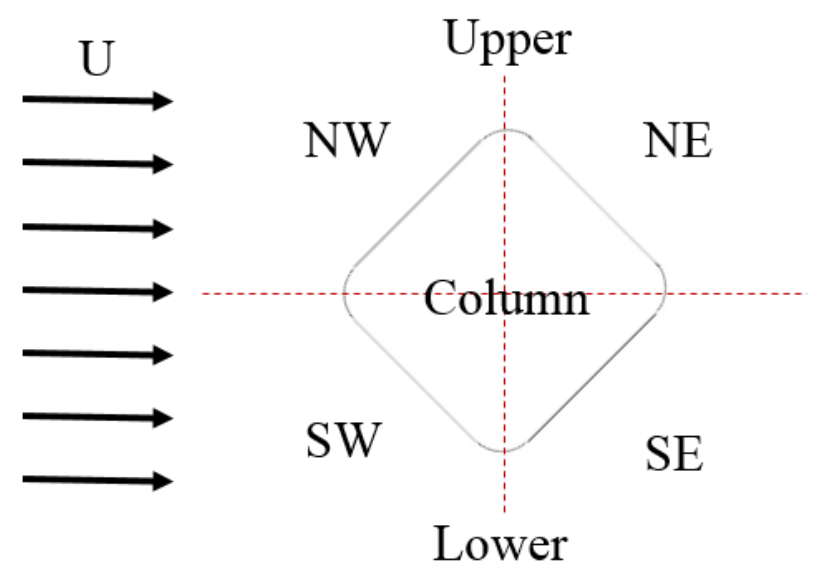

542 Fig. 30. Definition of the regions around the individual column.

543 Table 6. The chronological order of vortices genesis for each column.

\begin{tabular}{c|c|c}
\hline Column & Shear layer & Vortex street \\
\hline \multirow{2}{*}{1} & Upper & A1, A2 $\ldots$ \\
\cline { 2 - 3 } & Lower & B1, B2 $\ldots$ \\
\hline \multirow{2}{*}{2} & Upper & C1, C2 $\ldots$ \\
\cline { 2 - 3 } 3 & Lower & D1, D2 $\ldots$ \\
\cline { 2 - 3 } & Upper & E1, E2 $\ldots$ \\
\hline \multirow{2}{*}{4} & Lower & F1, F2 $\ldots$ \\
\cline { 2 - 3 } & Upper & $\mathrm{H} 1, \mathrm{G} 2 \ldots$ \\
\hline
\end{tabular}




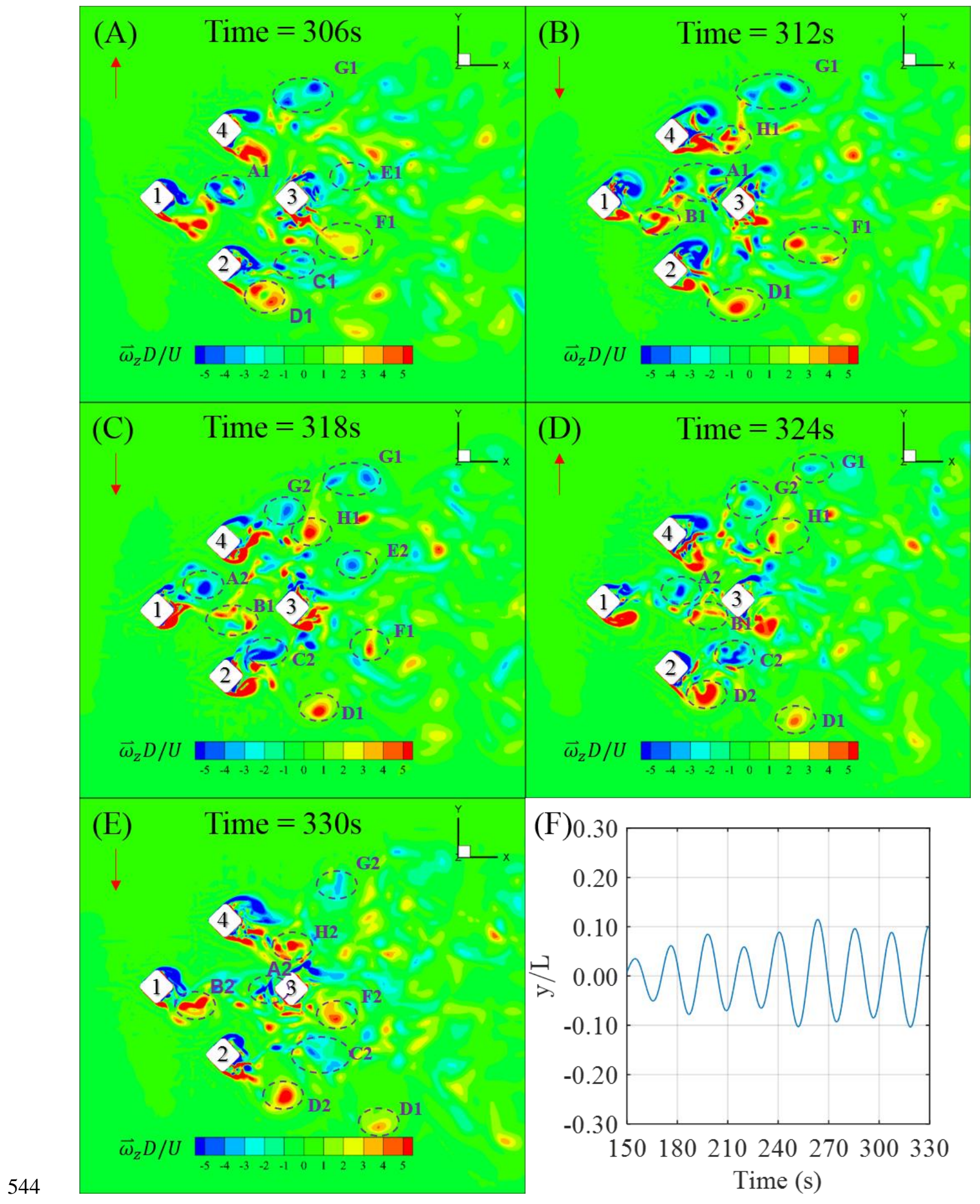

545 Fig. 31. A time series of the non-dimensional spanwise vorticity $\left(\vec{\omega}_{z} D / U\right)$ contours around the DDS at 546 middle draft showing the instantaneous flow fields around the DDS at $U r=3.9(\mathrm{~A}, \mathrm{~B}, \mathrm{C}, \mathrm{D}, \mathrm{E})$ and 
547 the non-dimensional transverse motion $(y / L)$ time history $(\mathrm{F})$; the red arrow is the DDS transverse

548 velocity direction.
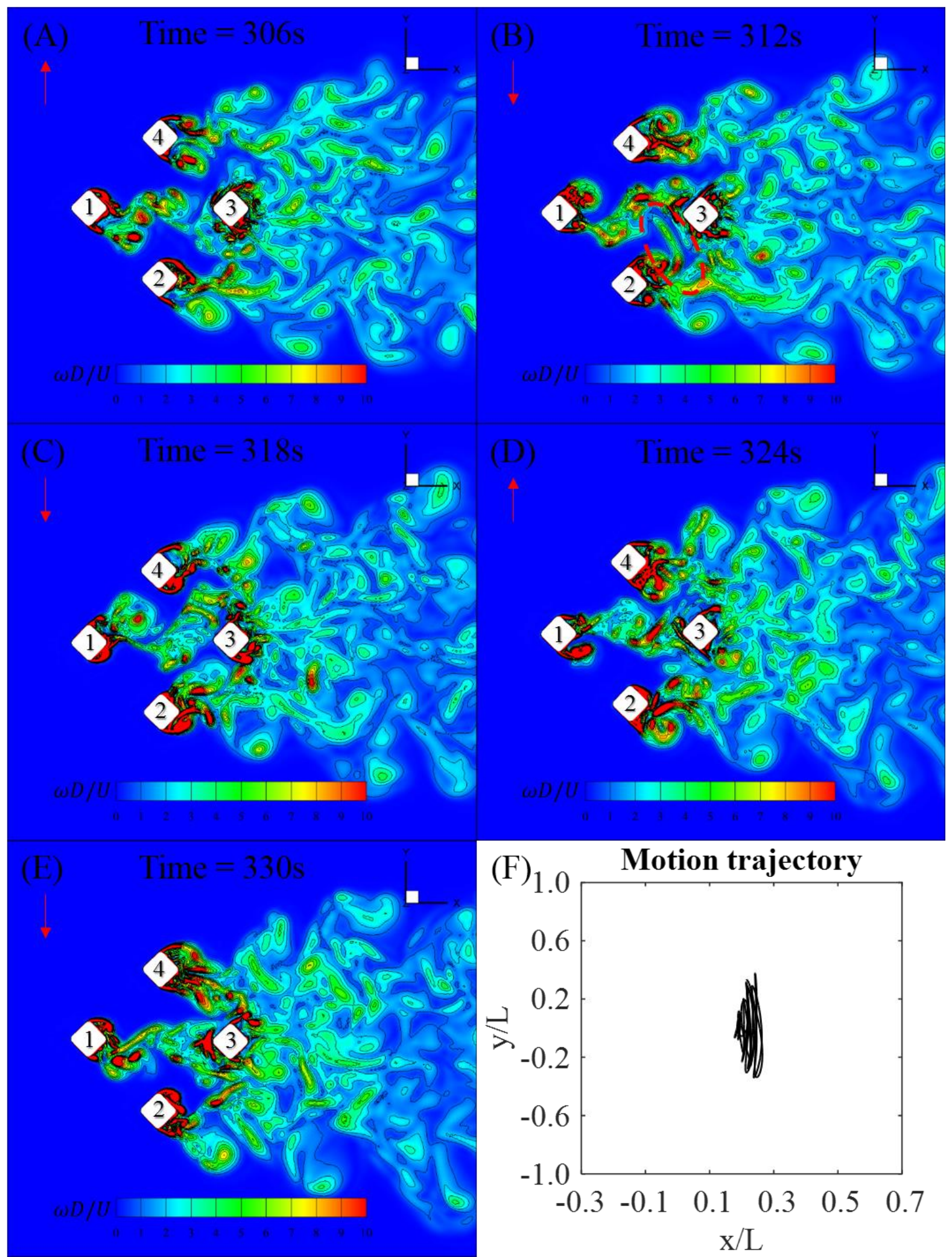
550 Fig. 32. A time series of the non-dimensional vorticity $(\omega D / U)$ contours around the DDS at middle 551 draft showing the instantaneous flow fields around the DDS at $U r=3.9(\mathrm{~A}, \mathrm{~B}, \mathrm{C}, \mathrm{D}, \mathrm{E})$ and the non552 dimensional motion trajectory $(\mathrm{F})$; the red arrow is the DDS transverse velocity direction.

553 Fig. 31 presents the non-dimensional spanwise vorticity $\left(\vec{\omega}_{z} D / U\right)$ contours at $U r=3.9$. As can be 554 seen, the vortices shed from the upstream column (Column 1) directly impinge on the downstream 555 column (Column 3) and then join into the downstream column's weak region. The vortex street can be 556 clearly found behind the upstream column where the " $2 \mathrm{~S}$ " type shedding occurs. Additionally, the 557 vortices shed from the portside and starboard side columns (Column 2 and 4) also impinge on the 558 downstream column, which are red circled in Fig. 32(B). These vortex patterns are not visible in the 559 spanwise vorticity contour (Fig. 31) indicating that there are three-dimensional effects on the flow 560 characteristics especially on the side columns' wake region and the flow region in front of the 561 downstream column. 


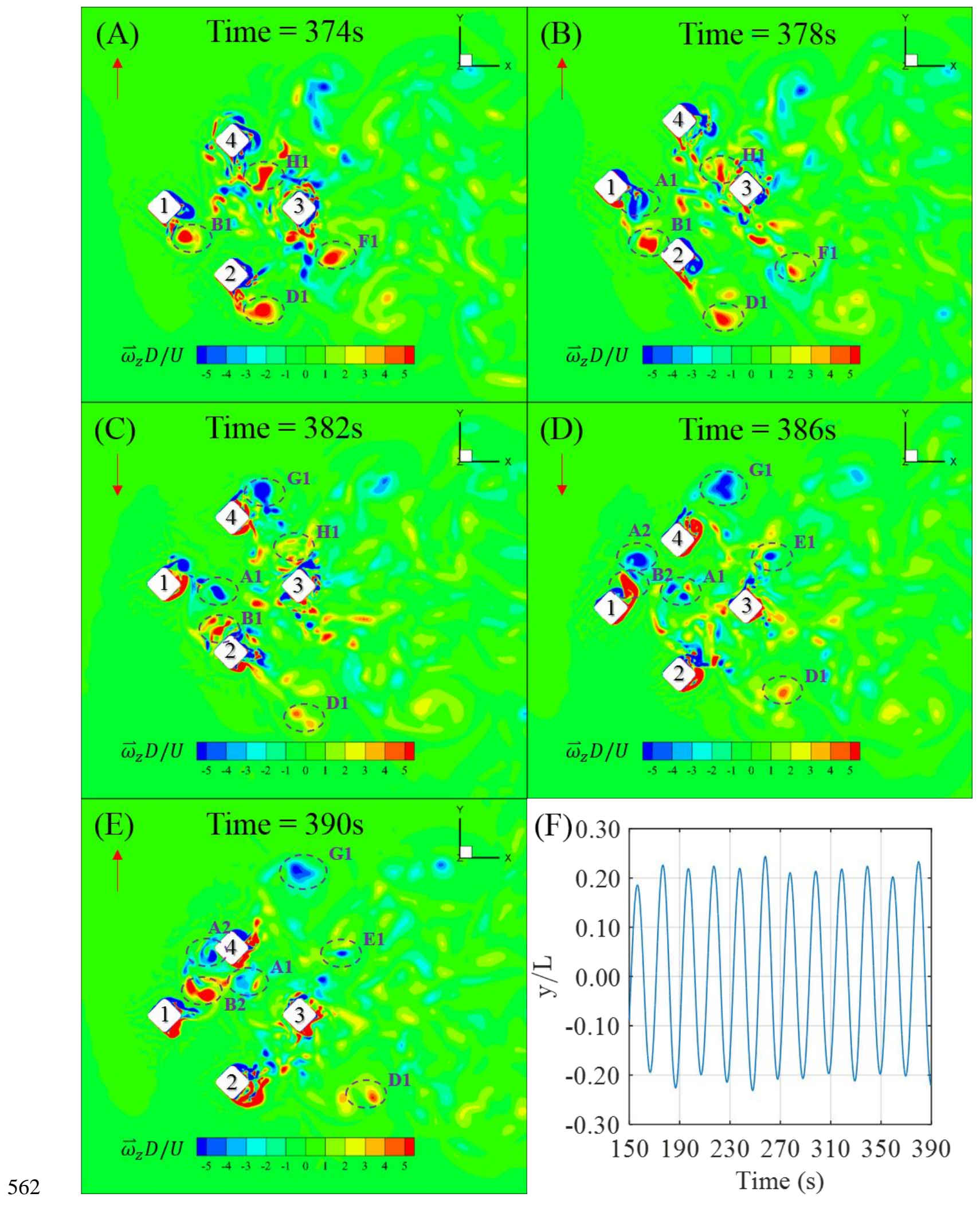

563 Fig. 33. A time series of the non-dimensional spanwise vorticity $\left(\vec{\omega}_{z} D / U\right)$ contours around the DDS at

564 middle draft showing the instantaneous flow fields around the DDS at $U r=5.1(\mathrm{~A}, \mathrm{~B}, \mathrm{C}, \mathrm{D}, \mathrm{E})$ and 
565 the non-dimensional transverse motion $(y / L)$ time history $(\mathrm{F})$; the red arrow is the DDS transverse

566 velocity direction.
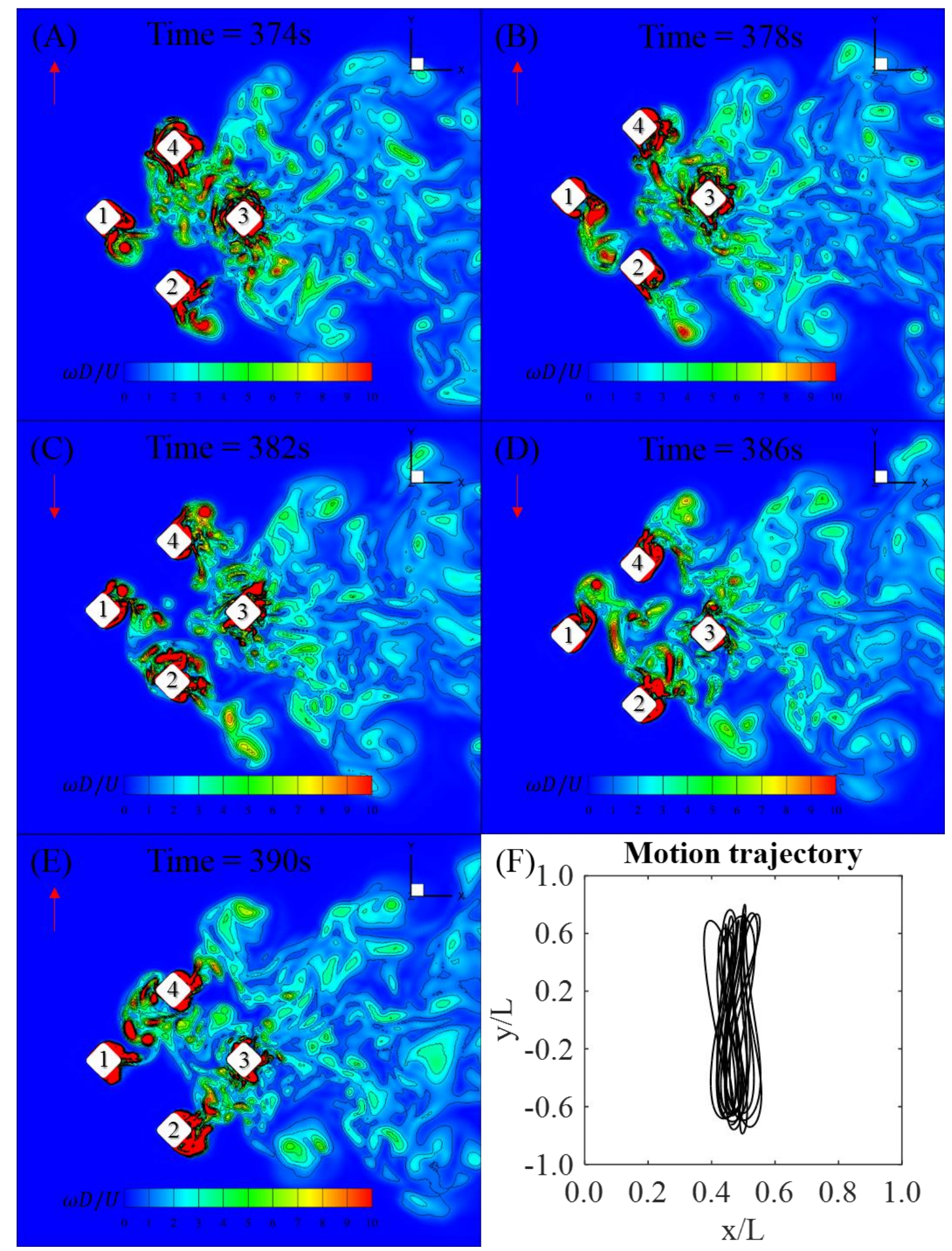
Fig. 34. A time series of the non-dimensional vorticity $(\omega D / U)$ contours around the DDS at middle

569 draft showing the instantaneous flow fields around the DDS at $U r=5.1$ (A, B, C, D, E) and the non-

570 dimensional motion trajectory $(\mathrm{F})$; the red arrow is the DDS transverse velocity direction.

571 With the increase of the $U r$, in the "lock-in" region, the flow patterns are changed. When $U r=5.1$, the structure oscillation frequency is close to the natural frequency of the structure in still water. As the result of the resonance developing, the motion starts to amplify and the flow patterns are changed significantly. Vortex streets only appear on the opposite of the transverse velocity direction (see Fig. 33 and Fig. 34). The vortices shed from the upstream column (Column 1) where the "P+S" type shedding occurred periodically and symmetrically impinge on the portside and starboard side (Column 2 and 4). Respectively, the vortices generated by the upstream column impinge on the NW face of the portside column (Column 2) and the SW face of the starboard side column (Column 4). Only one strong vortex will form on the opposite side to the transverse velocity direction behind portside and starboard side columns (see "D1, H1, G1" in Fig. 33). Also, the vortices shed from the

581 side columns impinge on the downstream column (Column 3). As a result, there is no clear vortex

582 street behind the downstream column. Small vortices in piece can be seen in the downstream of

583 Column 3. In addition, the motion trajectory shows a figure " 8 " shape under $U r=5.1$. 


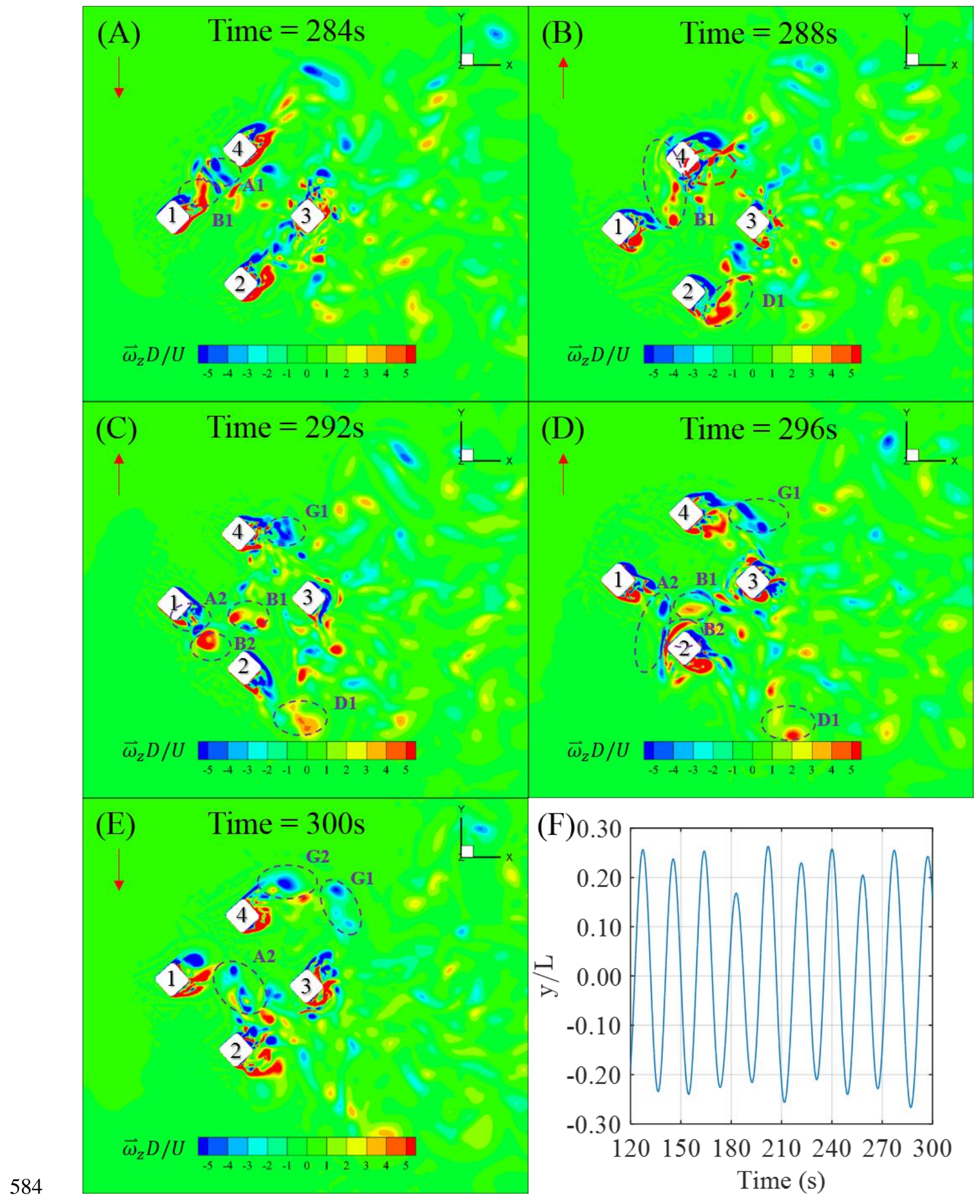

585 Fig. 35. A time series of the non-dimensional spanwise vorticity $\left(\vec{\omega}_{z} D / U\right)$ contours around the DDS at 586 middle draft showing the instantaneous flow fields around the DDS at $U r=6.6(\mathrm{~A}, \mathrm{~B}, \mathrm{C}, \mathrm{D}, \mathrm{E})$ and 
587 the non-dimensional transverse motion $(y / L)$ time history $(\mathrm{F})$; the red arrow is the DDS transverse

588 velocity direction.
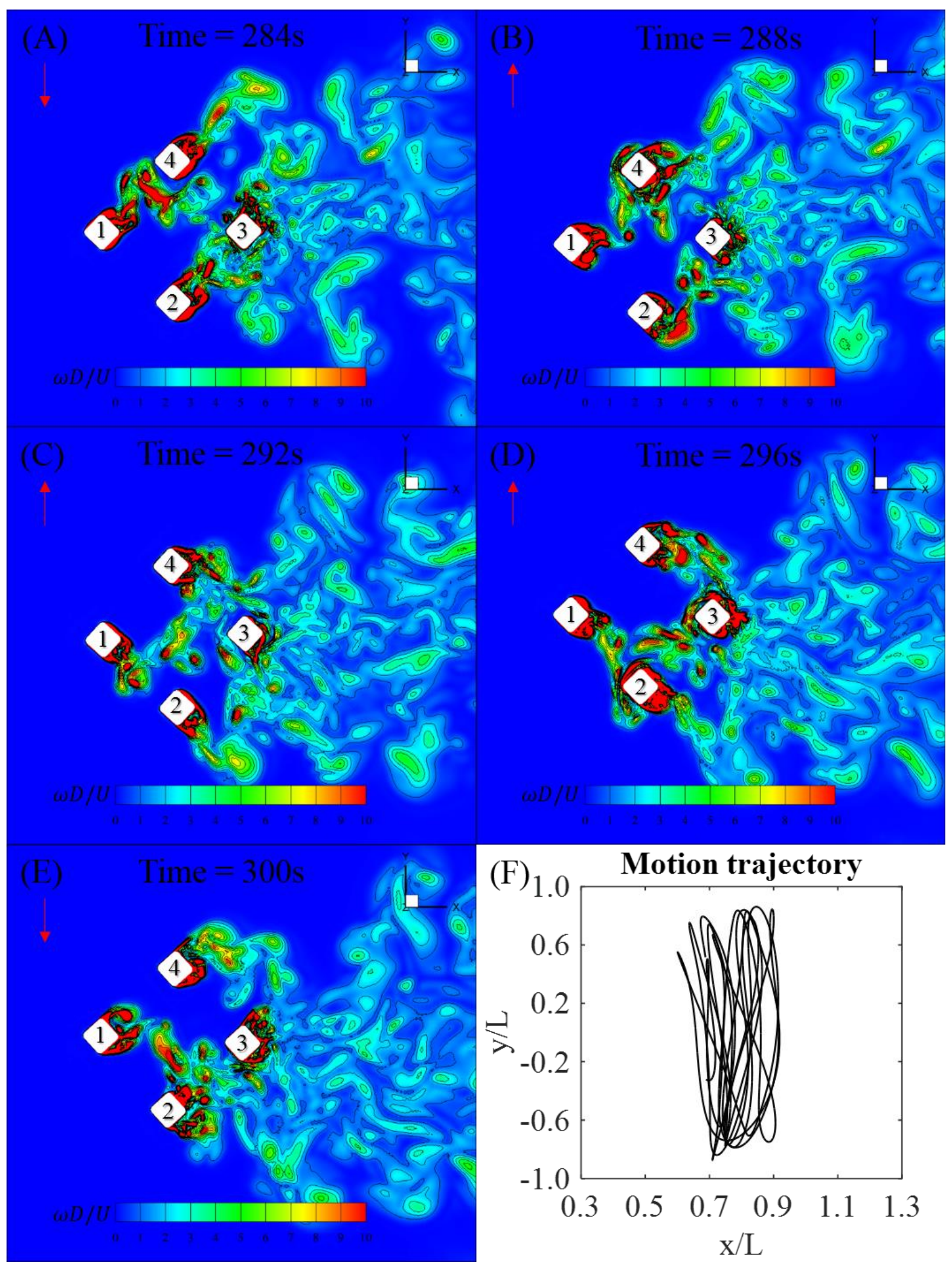
590 Fig. 36. A time series of the non-dimensional vorticity $(\omega D / U)$ contours around the DDS at middle

591 draft showing the instantaneous flow fields around the DDS at $U r=6.6(\mathrm{~A}, \mathrm{~B}, \mathrm{C}, \mathrm{D}, \mathrm{E})$ and the non-

592 dimensional motion trajectory $(\mathrm{F})$; the red arrow is the DDS transverse velocity direction.

593 With a further slight increase of the $U r$, the transverse motion keeps amplifying. However, the lift 594 force coefficient reduces (see Fig. 16 and Fig. 20). This hysteresis phenomenon can be explained by 595 Fig. 35. As the transverse motion is amplified, after impinging on the starboard side column (Column 596 4), the vortices that are shed from the upstream column (Column 1) move back to impinge on the 597 portside column (Column 2). This can be seen by following the trajectory of the vortices "B1".

598 Additionally, the vortices like "B1" affect the vortices detached from the upper side of Column 2 and 599 lower side of Column 4. As can be seen in Fig. 35(B) (red circled), two different clockwise vortices

600 are mixed together on the SE face of the starboard side column. The mixing of the vortices can 601 decrease the lift force on the structure. This is one of the reasons that makes the lift force coefficient on the structures drops while the transverse motion increases. By comparing the differences between

603 Fig. 33 and Fig. 35, there is another factor which may contribute to the hysteresis phenomenon. In

604 Fig. 35, it is seen that strong vortices are detached from both portside and starboard side at same time.

605 While in Fig. 33, only one strong vortex will form on the opposite side to the transverse velocity

606 direction behind portside and starboard side columns. The differences of the flow characteristics

607 shown in Fig. 33 and Fig. 35 lead to the peak point in the force domain occurs slightly earlier than 608 that in the transverse motion domain. 


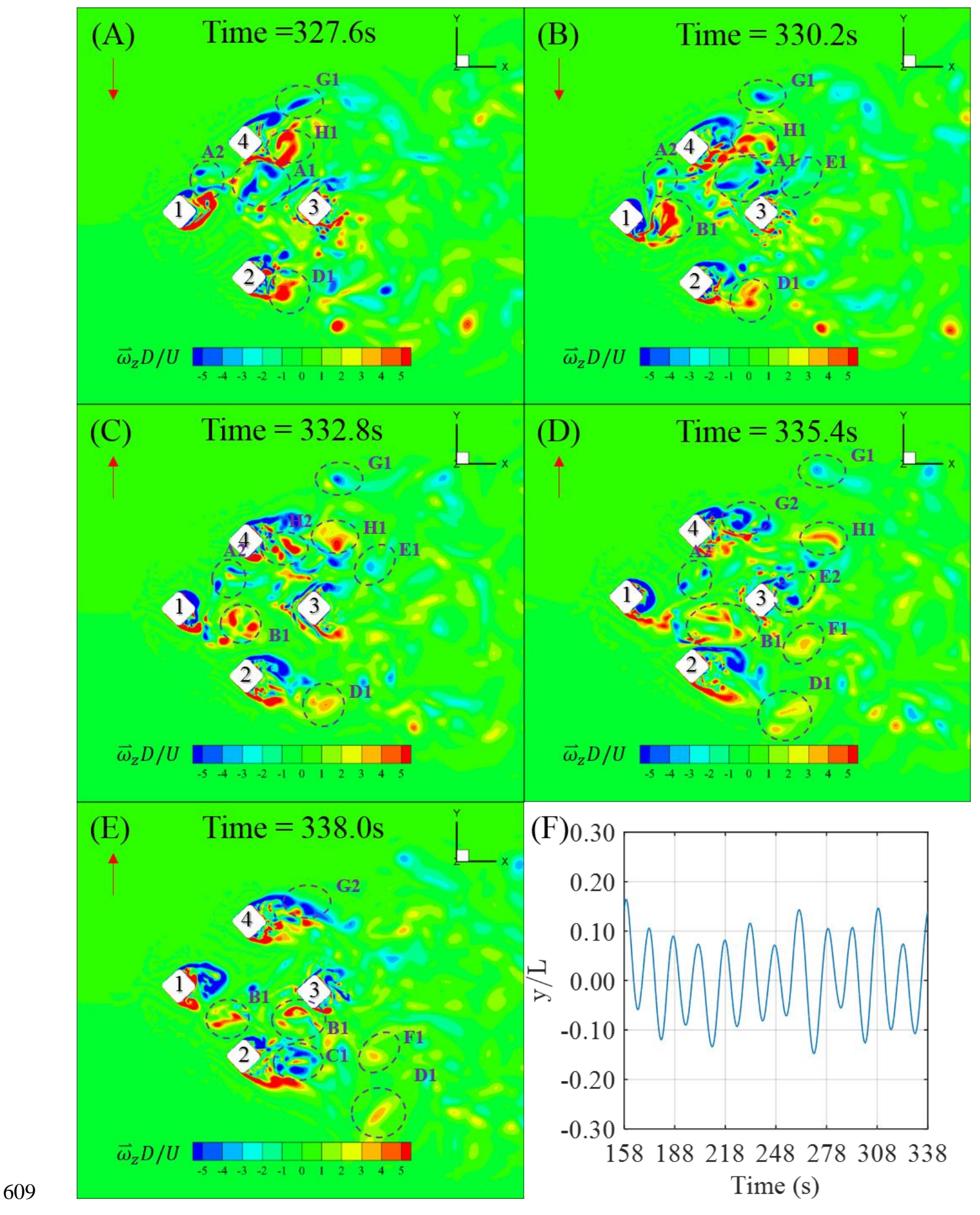

610 Fig. 37. A time series of the non-dimensional spanwise vorticity $\left(\vec{\omega}_{z} D / U\right)$ contours around the DDS at 611 middle draft showing the instantaneous flow fields around the DDS at $U r=8.9(\mathrm{~A}, \mathrm{~B}, \mathrm{C}, \mathrm{D}, \mathrm{E})$ and 
612 the non-dimensional transverse motion $(y / L)$ time history $(\mathrm{F})$; the red arrow is the DDS transverse

613 velocity direction.
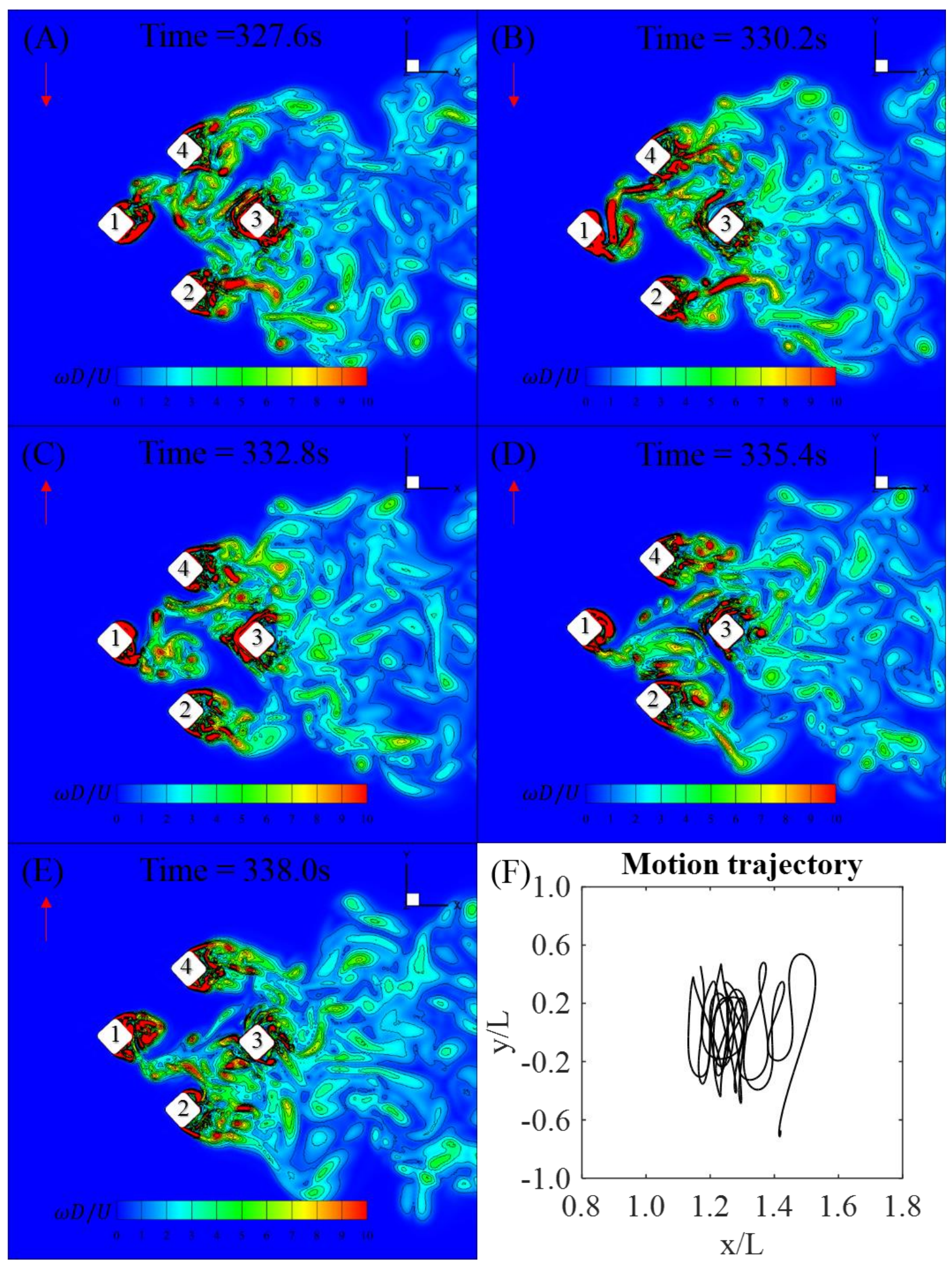
615 Fig. 38. A time series of the non-dimensional vorticity $(\omega D / U)$ contours around the DDS at middle

616 draft showing the instantaneous flow fields around the DDS at $U r=8.9(\mathrm{~A}, \mathrm{~B}, \mathrm{C}, \mathrm{D}, \mathrm{E})$ and the non-

617 dimensional motion trajectory $(\mathrm{F})$; the red arrow is the DDS transverse velocity direction.

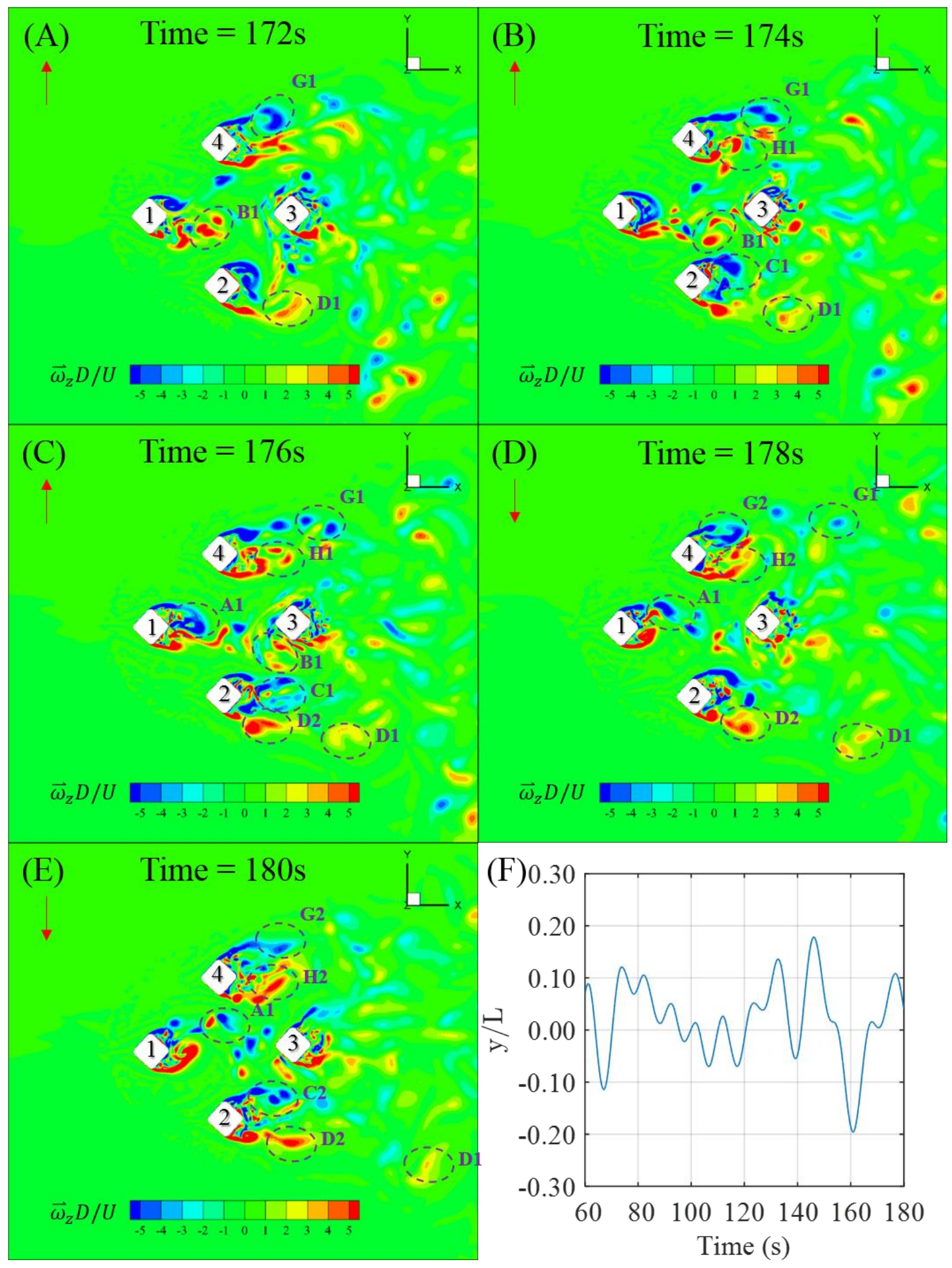


619 Fig. 39. A time series of the non-dimensional spanwise vorticity $\left(\vec{\omega}_{z} D / U\right)$ contours around the DDS at 620 middle draft showing the instantaneous flow fields around the DDS at $U r=12.1(\mathrm{~A}, \mathrm{~B}, \mathrm{C}, \mathrm{D}, \mathrm{E})$ and 621 the non-dimensional transverse motion $(y / L)$ time history $(\mathrm{F})$; the red arrow is the DDS transverse 622 velocity direction. 


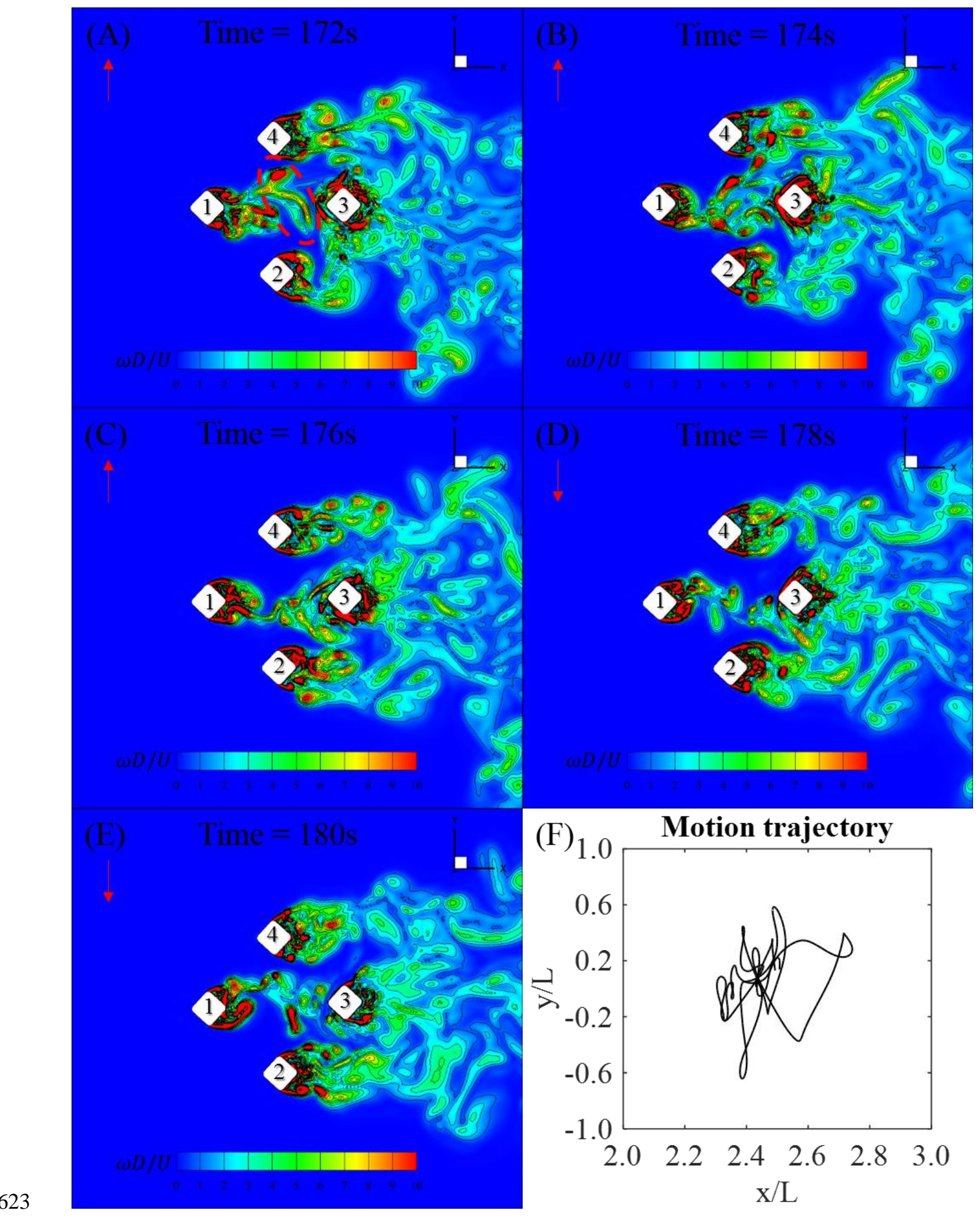

624 Fig. 40. A time series of the non-dimensional vorticity $(\omega D / U)$ contours around the DDS at middle

625 draft showing the instantaneous flow fields around the DDS at $U r=12.1$ (A, B, C, D, E) and the non-

626 dimensional motion trajectory (F); the red arrow is the DDS transverse velocity direction. 
627 When the reduced velocity reaches the "post lock-in" region, the vortices shed from the upstream 628 column (Column 1) no longer impinge on the incidence flow faces of the portside and starboard side 629 columns (Column 2 and 4). Instead, the vortices are seen to join the weak region of the portside and 630 starboard side (see Fig. 37 to Fig. 40). The vortex street behind the Column 1, 2 and 4 can be clearly 631 seen. In addition, it can be seen in Fig. 40(A) that parts of these vortices (red circled) do act on the incidence flow face of the downstream column (Column 3). As the vortices shed from the upstream column do not impinge on the portside and starboard side columns, the lift force coefficient and the transverse motion decrease and then remain a stable value in the measurement range of the "post lockin" region in the present study.

\section{Conclusions}

This paper presented a numerical study on the forces and VIM of a deep-draft semi-submersible.

Two different models were considered, i.e., a stationary model and a VIM model. For the stationary model, the drag and lift force distributions on each structural member of the DDS are discussed and followed by the flow pattern analyses. The vortex interactions between each column are presented to explain the hysteresis phenomenon. The numerical model predicts forces well compared to the experimental results. For the VIM model, the motion and force on the whole structures are analysed, and the "pre lock-in", "lock-in" and "post lock-in" phases can be accurately predicted in the present study. It is revealed that the discrepancies in the drag and lift forces between the numerical predictions and the experimental measurements at low reduced velocity is likely to be caused by the uncertainty in the experimental measurements at very low towing speed in the experiments. It is demonstrated that the numerical approach is a good way to predict the VIM responses at the low reduce velocity range.

Analysis of the drag and lift force coefficients on and the work done by different members of the DDS revealed that the portside and starboard side columns are the key structure members responsible for amplifying the VIM responses while the pontoons are acting to restrain VIM responses. 
652 The present numerical study confirmed the hysteresis phenomenon - the peak lift force occurs slightly

653 earlier than the peak transverse motion. By examining the flow patterns at the time instantaneous near

654 the peak response, it is revealed that the hysteresis phenomenon between the force and motion is

655 mainly due to the vortices shed from the upstream column move back to impinge on one of the side

656 columns after impinging on the other side column and the symmetrical strong vortices which shed

657 from the side columns.

658 This study focuses on the 45 degree flow incidence on the DDS, more incidences should be

659 considered and examined in order to obtain a more generalized understanding on VIM of a multi-

660 column structures.

661 Acknowledgment

662 The authors would like to acknowledge the support of Newton Fund of Royal Academy of

663 Engineering UK (NRCP/1415/211) and the National Natural Science Foundation of China (Grant No.

664 51279104). This work made use of the facilities of N8 HPC Centre of Excellence, provided and

665 funded by the N8 consortium and EPSRC (Grant No. EP/K000225/1).

\section{References}

667 Antony, A., Vinayan, V., Halkyard, J., Kim, S.-J., Holmes, S., Spernjak, D., 2015. A CFD Based Analysis of the Vortex Induced Motion of Deep-Draft Semisubmersibles, The Twenty-fifth International Offshore and Polar Engineering Conference. International Society of Offshore and Polar Engineers.

Antony, A., Vinayan, V., Madhavan, S., Parambath, A., Halkyard, J., Sterenborg, J., Holmes, S., Spernjak, D., Kim, S.J., Head, W., 2016. VIM Model Test of Deep Draft Semisubmersibles 
Celik, I.B., Ghia, U., Roache, P.J., 2008. Procedure for estimation and reporting of uncertainty due to discretization in CFD applications. Journal of fluids Engineering-Transactions of the ASME $130(7)$.

Chen, J.M., Chiou, C.-C., 1997. Flow past a blunt flat plate subjected to the disturbance of incident vortex street. Journal of Wind Engineering and Industrial Aerodynamics 66 (3), 179-196.

Fujarra, A.L.C., Rosetti, G.F., de Wilde, J., Gonçalves, R.T., 2012. State-of-art on vortex-induced motion: a comprehensive survey after more than one decade of experimental investigation, ASME 2012 31st International Conference on Ocean, Offshore and Arctic Engineering. American Society of Mechanical Engineers, pp. 561-582.

Gonçalves, R.T., Fujarra, A.L.C., Rosetti, G.F., Kogishi, A.M., Koop, A., 2015. Effects of Column Designs on the VIM Response of Deep-Draft Semi-Submersible Platforms, The Twenty-fifth International Offshore and Polar Engineering Conference. International Society of Offshore and Polar Engineers.

Gonçalves, R.T., Rosetti, G.F., Fujarra, A.L.C., Oliveira, A.C., 2012. Experimental study on vortexinduced motions of a semi-submersible platform with four square columns, Part I: Effects of current incidence angle and hull appendages. Ocean Engineering 54, 150-169.

Gonçalves, R.T., Rosetti, G.F., Fujarra, A.L.C., Oliveira, A.C., 2013. Experimental study on vortexinduced motions of a semi-submersible platform with four square columns, Part II: Effects of surface waves, external damping and draft condition. Ocean Engineering 62, 10-24.

Hong, Y., Choi, Y., Lee, J., Kim, Y., 2008. Vortex-induced motion of a deep-draft semi-submersible in current and waves, The Eighteenth International Offshore and Polar Engineering Conference. International Society of Offshore and Polar Engineers.

Kokkinis, T., Sandström, R.E., Jones, H.T., Thompson, H.M., Greiner, W.L., 2004. Development of a Stepped Line Tensioning Solution for Mitigating VIM Effects in Loop Eddy Currents for the Genesis Spar, ASME 2004 23rd International Conference on Offshore Mechanics and Arctic Engineering. American Society of Mechanical Engineers, pp. 995-1004. 
Koop, A., Rijken, O., Vaz, G., Maximiano, A., Rosetti, G., 2016. CFD Investigation on Scale and Damping Effects for Vortex Induced Motions of a Semi-Submersible Floater, Offshore Technology Conference. Offshore Technology Conference.

Lam, K., Li, J.Y., So, R.M.C., 2003b. Force coefficients and Strouhal numbers of four cylinders in cross flow. Journal of Fluids and structures 18 (3), 305-324.

Lee, S.-K., Chien, H.-P., Gu, H., 2014. CFD Study of Deep Draft SemiSubmersible VIM, Offshore Technology Conference-Asia. Offshore Technology Conference.

Lefevre, C., Constantinides, Y., Kim, J.W., Henneke, M., Gordon, R., Jang, H., Wu, G., 2013. Guidelines for CFD Simulations of Spar VIM, ASME 2013 32nd International Conference on Ocean, Offshore and Arctic Engineering. American Society of Mechanical Engineers, pp. V007T008A019-V007T008A019.

Liang, Y., Tao, L., Xiao, L., Liu, M., 2016. Experimental and Numerical Study on Vortex-Induced Motions of a Deep-Draft Semi-Submersible, Manuscript submitted for publication.

Liu, C.-H., Chen, J.M., 2002. Observations of hysteresis in flow around two square cylinders in a tandem arrangement. Journal of Wind Engineering and Industrial Aerodynamics 90 (9), 10191050.

Liu, M., Xiao, L., Lyu, H., Tao, L., 2015. Numerical Analysis of Pontoon Effect on Flow-Induced Forces of the Deep Draft Semisubmersible in a Cross-Flow, ASME 2015 34th International Conference on Ocean, Offshore and Arctic Engineering. American Society of Mechanical Engineers, pp. V001T001A030-V001T001A030.

Ljungkrona, L., Norberg, C.H., Sunden, B., 1991. Free-stream turbulence and tube spacing effects on surface pressure fluctuations for two tubes in an in-line arrangement. Journal of Fluids and structures 5 (6), 701-727.

Ma, W., Wu, G., Thompson, H., Prislin, I., Maroju, S., 2013. Vortex induced motions of a column stabilized floater, Proceedings of the DOT International Conference, pp. 22-24.

Magee, A., Sheikh, R., Guan, K.Y.H., Choo, J.T.H., Malik, A.M.A., Ghani, M.P.A., Abyn, H., 2011. Model tests for VIM of multi-column floating platforms, ASME 2011 30th International 

Engineers, pp. 127-136.

Rijken, O., Leverette, S., 2008. Experimental Study into Vortex Induced Motion Response of Semi Submersibles with Square Columns, ASME 2008 27th International Conference on Offshore Mechanics and Arctic Engineering. American Society of Mechanical Engineers, pp. 263-276.

Rijken, O., Leverette, S., 2009. Field measurements of vortex induced motions of a deep draft semisubmersible, ASME 2009 28th International Conference on Ocean, Offshore and Arctic Engineering. American Society of Mechanical Engineers, pp. 739-746.

Rijken, O., Schuurmans, S., Leverette, S., 2011. Experimental investigations into the influences of SCRs and appurtenances on DeepDraft Semisubmersible Vortex Induced Motion response, Proceedings of the 30th International Conference on Ocean, Offshore and Arctic Engineering, Rotterdam, The Netherlands, OMAE2011-49365.

Sarpkaya, T., 2004. A critical review of the intrinsic nature of vortex-induced vibrations. Journal of Fluids and structures 19 (4), 389-447.

Schewe, G., 1983. On the force fluctuations acting on a circular cylinder in crossflow from subcritical up to transcritical Reynolds numbers. Journal of Fluid Mechanics 133, 265-285.

Shur, M.L., Spalart, P.R., Strelets, M.K., Travin, A.K., 2008. A hybrid RANS-LES approach with delayed-DES and wall-modelled LES capabilities. International Journal of Heat and Fluid Flow 29 (6), 1638-1649.

Spalart, P.R., Jou, W.H., Strelets, M., Allmaras, S.R., 1997. Comments on the feasibility of LES for wings, and on a hybrid RANS/LES approach. Advances in DNS/LES 1, 4-8.

Tan, J.H.C., Magee, A., Kim, J.W., Teng, Y.J., Zukni, N.A., 2013. CFD Simulation for Vortex Induced Motions of a Multi-Column Floating Platform, ASME 2013 32nd International Conference on Ocean, Offshore and Arctic Engineering. American Society of Mechanical Engineers, pp. V007T008A066-V007T008A066.

Vikestad, K., Vandiver, J.K., Larsen, C.M., 2000. Added mass and oscillation frequency for a circular cylinder subjected to vortex-induced vibrations and external disturbance. Journal of Fluids and structures 14 (7), 1071-1088. 
Vinayan, V., Antony, A., Halkyard, J., Kim, S.-J., Holmes, S., Spernjak, D., 2015. Vortex-Induced Motion of Deep-Draft Semisubmersibles: A CFD-Based Parametric Study, ASME 2015 34th International Conference on Ocean, Offshore and Arctic Engineering. American Society of Mechanical Engineers, pp. V002T008A003-V002T008A003.

Waals, O.J., Phadke, A.C., Bultema, S., 2007. Flow Induced Motions on Multi Column Floaters, ASME 2007 26th International Conference on Offshore Mechanics and Arctic Engineering. American Society of Mechanical Engineers, pp. 669-678.

Zhang, H., Yang, J., Xiao, L., Lu, H., 2014. Study on added mass coefficient and oscillation frequency for a Truss Spar subjected to Vortex-Induced Motions. Ships and Offshore Structures 9 (1), 54-63. 


\section{List of Tables}

770 Table 1. Main characteristics of the DDS unit (The model I is the stationary model which presents

771 scale ratio as 1:128, and the model II is the VIM model which presents scale ratio as 1:64).

772 Table 2. Calculations of discretization error (Celik et al., 2008); GCI index represents the numerical 773 uncertainty.

774 Table 3. Summary of the various studies on VIM of the multiple square section shaped columns 775 structures.

776 Table 4. The resulting force coefficient values $\bar{C}_{D}, C_{\text {Lrms }}$ and $S t$ for the flow over a stationary DDS.

777 Table 5. Natural periods of the motions in calm water.

778 Table 6. The chronological order of vortices genesis for each column. 


\section{List of Figures}

781 Fig. 1. The DDS model (A is the entire model and B is the decomposed model which show the 782 definition of the individual members).

783 Fig. 2. Computational domain.

784 Fig. 3. Visualisation of the mesh at the middle draft level of the DDS ( $X Y$ plane at the middle draft of 785 the DDS).

786 Fig. 4. Calculations with additional grid refinements for the Strouhal number $(S t)$.

787 Fig. 5. Non-dimensional transverse characteristic amplitudes $\left(A_{y} / L\right)$ obtained from the present towing 788 tank test.

Fig. 6. Non-dimensional spanwise vorticity $\left(\vec{\omega}_{z} D / U\right)$ contours around the DDS at middle draft showing the flow fields when "lock-in" has occurred (A: $U r=6.4,0$ degree incidence. B: $U r=6.6,45$

791 degree incidence).

792 Fig. 7. Mean drag coefficient $\left(\bar{C}_{D}\right)$ from the numerical and experimental results for the stationary 793 model.

Fig. 8. Root mean square lift coefficient $\left(C_{\text {Lrms }}\right)$ from the numerical and experimental results for the stationary model.

Fig. 9. Strouhal number (St) from the numerical and experimental results for the stationary model.

797 Fig. 10. Mean drag coefficients $\left(\bar{C}_{D}\right)$ on each member of the stationary DDS.

Fig. 11. Root mean square lift coefficients $\left(C_{\text {Lrms }}\right)$ on each member of the stationary DDS. 
Fig. 12. Mean lift coefficient $\left(\bar{C}_{L}\right)$ on each member of the stationary DDS.

800 Fig. 13. Lift force coefficient time history on different members of the DDS at $R e=4.3 \times 10^{4}$,

801 including locally zoomed in the last $6 \mathrm{~s}$.

802 Fig. 14. A time series of the pressure distribution around the DDS at middle draft showing the 803 instantaneous flow fields around the DDS at $R e=4.3 \times 10^{4}$ corresponding to the lift force coefficient 804 time history (A: 120.6s; B: 122.4s; C: 124.2s; D: 126.0s).

Fig. 15. A time series of non-dimensional spanwise vorticity $\left(\vec{\omega}_{z} D / U\right)$ contours around the DDS at the middle draft level showing the instantaneous flow fields around the DDS at $R e=4.3 \times 10^{4}$ corresponding to the lift force coefficient time history (A: 120.6s; B: 122.4s; C: 124.2s; D: 126.0s). Fig. 16. Non-dimensional transverse characteristic amplitudes $\left(A_{y} / L\right)$, the $U r$ is defined based on the natural period of the transverse motion. natural period of the transverse motion.

Fig. 18. Non-dimensional yaw characteristic amplitudes, the $U r$ is defined based on the natural period 813 of the yaw motion.

814 Fig. 19. Mean drag coefficient $\left(\bar{C}_{D}\right)$ from the numerical and experimental results on the VIM model, 815 the $U r$ is defined based on the natural period of the transverse motion.

816 Fig. 20. Root mean square lift coefficient $\left(C_{\text {Lrms }}\right)$ from the numerical and experimental results on the 817 VIM model, the $U r$ is defined based on the natural period of the transverse motion.

818 Fig. 21. Added mass coefficient $\left(C_{a}\right)$ of the VIM model from the numerical predictions and the 819 experiments, the $U r$ is defined based on the natural period of the transverse motion. 
820 Fig. 22. FFT of the transverse motions and the lift force coefficients at $U r=3.9$, (a) transverse

821 motion; (b) lift force coefficient.

822 Fig. 23. FFT of the transverse motions and the lift force coefficients at $U r=5.1$, (a) transverse 823 motion; (b) lift force coefficient.

824 Fig. 24. FFT of the transverse motions and the lift force coefficients at $U r=6.6$, (a) transverse 825 motion; (b) lift force coefficient.

826 Fig. 25. FFT of the transverse motions and the lift force coefficients at $U r=8.9$, (a) transverse 827 motion; (b) lift force coefficient.

828 Fig. 26. FFT of the transverse motions and the lift force coefficients at $U r=12.1$, (a) transverse 829 motion; (b) lift force coefficient.

830 Fig. 27. Mean drag coefficients $\left(\bar{C}_{D}\right)$ on each member of the DDS from the VIM model.

831 Fig. 28. Root mean square lift coefficients $\left(C_{\text {Lrms }}\right)$ on each member of the DDS from the VIM model.

832 Fig. 29. Work done by each member of the DDS on VIM model.

833 Fig. 30. Definition of the regions around the individual column.

834 Fig. 31. A time series of the non-dimensional spanwise vorticity $\left(\vec{\omega}_{z} D / U\right)$ contours around the DDS at 835 middle draft showing the instantaneous flow fields around the DDS at $U r=3.9(\mathrm{~A}, \mathrm{~B}, \mathrm{C}, \mathrm{D}, \mathrm{E})$ and 836 the non-dimensional transverse motion $(y / L)$ time history $(\mathrm{F})$; the red arrow is the DDS transverse 837 velocity direction. 
Fig. 32. A time series of the non-dimensional vorticity $(\omega D / U)$ contours around the DDS at middle draft showing the instantaneous flow fields around the DDS at $U r=3.9$ (A, B, C, D, E) and the nondimensional motion trajectory $(\mathrm{F})$; the red arrow is the DDS transverse velocity direction.

Fig. 33. A time series of the non-dimensional spanwise vorticity $\left(\vec{\omega}_{z} D / U\right)$ contours around the DDS at middle draft showing the instantaneous flow fields around the DDS at $U r=5.1$ (A, B, C, D, E) and the non-dimensional transverse motion $(y / L)$ time history $(\mathrm{F})$; the red arrow is the DDS transverse velocity direction.

Fig. 34. A time series of the non-dimensional vorticity $(\omega D / U)$ contours around the DDS at middle draft showing the instantaneous flow fields around the DDS at $U r=5.1$ (A, B, C, D, E) and the nondimensional motion trajectory (F); the red arrow is the DDS transverse velocity direction.

Fig. 35. A time series of the non-dimensional spanwise vorticity $\left(\vec{\omega}_{z} D / U\right)$ contours around the DDS at middle draft showing the instantaneous flow fields around the DDS at $U r=6.6(\mathrm{~A}, \mathrm{~B}, \mathrm{C}, \mathrm{D}, \mathrm{E})$ and the non-dimensional transverse motion $(y / L)$ time history $(\mathrm{F})$; the red arrow is the DDS transverse velocity direction.

Fig. 36. A time series of the non-dimensional vorticity $(\omega D / U)$ contours around the DDS at middle draft showing the instantaneous flow fields around the DDS at $U r=6.6$ (A, B, C, D, E) and the nondimensional motion trajectory $(\mathrm{F})$; the red arrow is the DDS transverse velocity direction.

Fig. 37. A time series of the non-dimensional spanwise vorticity $\left(\vec{\omega}_{z} D / U\right)$ contours around the DDS at middle draft showing the instantaneous flow fields around the DDS at $U r=8.9$ (A, B, C, D, E) and the non-dimensional transverse motion $(y / L)$ time history $(\mathrm{F})$; the red arrow is the DDS transverse velocity direction. 
859 Fig. 38. A time series of the non-dimensional vorticity $(\omega D / U)$ contours around the DDS at middle

860 draft showing the instantaneous flow fields around the DDS at $U r=8.9(\mathrm{~A}, \mathrm{~B}, \mathrm{C}, \mathrm{D}, \mathrm{E})$ and the non-

861 dimensional motion trajectory $(\mathrm{F})$; the red arrow is the DDS transverse velocity direction.

862 Fig. 39. A time series of the non-dimensional spanwise vorticity $\left(\vec{\omega}_{z} D / U\right)$ contours around the DDS at

863 middle draft showing the instantaneous flow fields around the DDS at $U r=12.1(\mathrm{~A}, \mathrm{~B}, \mathrm{C}, \mathrm{D}, \mathrm{E})$ and

864 the non-dimensional transverse motion $(y / L)$ time history $(F)$; the red arrow is the DDS transverse

865 velocity direction.

866 Fig. 40. A time series of the non-dimensional vorticity $(\omega D / U)$ contours around the DDS at middle

867 draft showing the instantaneous flow fields around the DDS at $U r=12.1$ (A, B, C, D, E) and the non-

868 dimensional motion trajectory $(\mathrm{F})$; the red arrow is the DDS transverse velocity direction. 